\title{
A mathematical model of water and nutrient transport in xylem vessels of a wheat plant
}

\author{
S. Payvandi ${ }^{a, b}$, K.R. Daly ${ }^{a, b}$, D.L. Jones ${ }^{d}$, P. Talboys ${ }^{d}$, K.C. Zygalakis ${ }^{c, b}$, T. Roose ${ }^{a, b}$
}

$a$ : Engineering Sciences, Faculty of Engineering and the Environment, University of Southampton, University Road, Southampton, SO17 1BJ,

UK

b: Crop Systems Engineering Group, Institute for Life Sciences, University of Southampton, University Road, Southampton, SO17 1BJ, UK c: Mathematical Sciences, Faculty of Social and Human Sciences, University of Southampton, University Road, Southampton, SO17 1DN, UK

$d$ : School of Environment, Natural Resources and Geography, University of Bangor, Bangor, Gwynedd, LL57 2UW, UK

Key words: vascular transport, phosphate, multidirectional flow, plant modelling.

\begin{abstract}
At a time of increasing global demand for food, dwindling land and resources, and escalating pressures from climate change, the farming industry is undergoing financial strain, with a need to improve efficiency and crop yields. In order to improve efficiencies in farming, and in fertiliser usage in particular, understanding must be gained of the fertiliser-to-crop-yield pathway. We model one aspect of this pathway; the transport of nutrients within the vascular tissues of a crop plant from roots to leaves. We present a mathematical model of the transport of nutrients within the xylem vessels in response to the evapotranspiration of water. We determine 7 different classes of flow, including positive unidirectional flow, which is optimal for nutrient transport from the roots to the leaves; and root multidirectional flow, which is similar to the hydraulic lift process observed in plants. We also investigate the effect of diffusion on nutrient transport and find that diffusion can be significant at the vessel termini especially if there is an axial efflux of nutrient, and at night when transpiration is minimal. Models such as these can then be coupled to whole-plant models to be used for optimisation of nutrient delivery scenarios.
\end{abstract}




\section{Introduction}

In crop plants such as wheat, fertilisers are often necessary to ensure high crop yields, especially at a time of farming intensification. Fertilisers are applied to soils which are then taken up by the root systems. Phosphate is a key fertiliser for crops, but is often the most limiting due to its low mobility in soils (Bucher, 2007). Phosphate plays a vital role in plant function; it is important for photosynthesis, respiration, energy conservation and the metabolism of carbon (Foyer \& Spencer, 1986); with the result that phosphate application significantly increases grain yield (Gallet et al., 2003). However, the rising global population, together with the disruptive effects of climate change, are putting increasing pressures on the agricultural industry to satisfy the growing demand for food (Cordell et al., 2009). Therefore there is a need to improve the efficiency of phosphate use (Bennett \& Elser, 2011), which requires understanding and improvement of all the processes in the fertiliser-to-crop-yield pathway. In this paper we consider the transport of phosphate and water from the roots to the leaves within an idealised model of the xylem vessels of a wheat plant. In the future, this model can be coupled to whole-plant models which can be used in conjunction with experimental data to determine and optimise plant parameters and functions such as phosphate uptake and loading. Note that this research is also applicable to nutrients which possess similar transport properties to phosphate, and hence we use the terms phosphate and nutrient interchangeably.

In angiosperms such as wheat, xylem tissue is comprised of lignified vessel elements resulting in a continuous, low-resistance pipe-like structure, and phosphate is transported within these vessels by advection caused by the flow of water. Water flows upwards within the xylem vessels as a passive consequence of evapotranspiration through the stomata in the leaves. During the day, the stomatal pores open, allowing the diffusion of carbon dioxide from the air to the mesophyll cells for photosynthesis, whilst water diffuses out of the leaf down the water vapour concentration gradient. This loss of water is known as transpiration and creates a negative potential in the leaf air-spaces that pulls water out of the xylem vessels and into the mesophyll cell walls. This consequently creates a gradient in pressure between the top of the xylem and the roots, pulling water upwards under tension. Water uptake from the soil to roots is also driven by the gradient in pressure between the xylem and the soil pore water. This transport mechanism is known as cohesion-tension theory and was proposed by Dixon \& Joly (1895). For a review consult Tyree (1997), Steudle (2001), and Kramer \& Boyer (1995).

In this paper we assume that the flow of water is driven only by the pressure difference between roots and leaves and consider the effect that gravity and varying leaf pressures have on the flow. For certain parameter regimes the flow of water can become multidirectional which would inhibit nutrient transport to the leaves. We therefore seek the conditions for unidirectional 
flow everywhere in the xylem vessel which would give rise to optimal nutrient transport to the leaves.

Diffusional transport of nutrients has generally been neglected in the literature as diffusion is small compared to convection in the main bulk flow. However, diffusion is significant near the vessel boundaries, especially when the convective transport falls to zero at the xylem termini in the root tips. In addition, diffusional transport is important throughout the vessels when the transpirational flow diminishes during the night. Finally, the inclusion of diffusion permits the modelling of an axial flux of nutrient through the xylem termini in the root tips, which may occur in response to the nutrient sink caused by the meristemic tissue at the growing root tip. For example Bingham \& Stevenson (1992) show that root tips of wheat consume more glucose compared to the rest of the root, and Bowen (1970) show that root tips of Pinus radiata uptake and retain more phosphate.

The convective and diffusional transport of nutrients within the vessels is balanced by the loading and unloading (which may be active and/or passive) of nutrient in response to the activity of the surrounding tissues. For example, phosphate enters the xylem vessel in the root zone, and exits in the stem and leaf zones. The majority of the phosphate is unloaded in the leaf region where it is used for photosynthesis. For simplicity, we assume that the loading is constant in the different plant zones, although the loading can easily be modified to incorporate more complicated functions, such as those that depend on internal concentration or axial distance.

In Section 2 we present the mathematical model which describes the flow of water and phosphate in the xylem vessels in response to a pressure difference between the roots and leaves. In Section 3 we discuss the values of the model parameters particular to phosphate transport in wheat and nondimensionalise the model. In Section 4 we analytically solve for the flow of water and determine the conditions for unidirectional flow and hence optimal flow of phosphate. In Section 5 we present analytical and numerical solutions of phosphate transport in the xylem vessel, and finally in Section 6 we discuss the implications of the model findings.

\section{Model}

We consider the transport of water and nutrients from roots to leaves in the xylem vessels of a small plant such as wheat. We follow a similar approach to the phloem model of Jensen et al. $(2011,2012)$ and separate the plant and xylem tissue into three zones in the $\hat{z}$ direction; the leaves $L$, the stem $S$, and the roots $R$ as shown in Figure 1. The leaf zone is represented by $0<\hat{z}<\hat{z}_{1}$, the stem zone is $\hat{z}_{1}<\hat{z}<\hat{z}_{2}$ and the root zone is $\hat{z}_{2}<\hat{z}<\hat{z}_{3}$ where $\hat{z}, \hat{z_{1}}, \hat{z_{2}}$, and $\hat{z_{3}}$ are measured in metres. We prescribe that the xylem tissue consists of $\hat{v}_{j}^{i}$ number of semipermeable cylindrical vessels of radius 
$\hat{a}_{j}^{i}(\mathrm{~m})$ where $j$ is an index for the radii for the different vessels and $i$ is an index for plant zone. We consider the transport of phosphate only, which we denote as $\hat{n}\left(\mathrm{~mol} \mathrm{~m}^{-3}\right)$. All symbols are given in Table 1 .

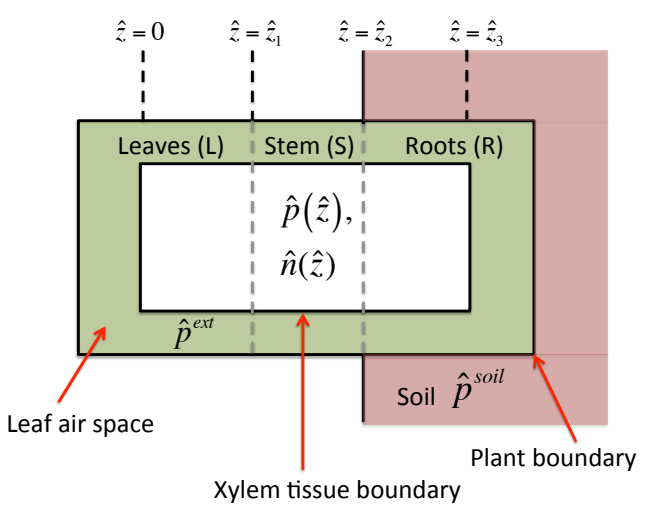

Figure 1: Model of the xylem within a plant. $\hat{z}=0$ is the top of the xylem in the leaf, $\hat{z}_{1}$ is the leaf-stem boundary, $\hat{z}_{2}$ is the stem-root boundary, and $\hat{z}_{3}$ is the terminus of the xylem in the root. The pressures in the soil $\left(\hat{p}^{\text {soil }}\right)$ and leaf air-spaces $\left(\hat{p}^{\text {ext }}\right)$ are constant, and the xylem pressure, $\hat{p}$, and nutrient concentration, $\hat{n}$, are functions of $\hat{z}$.

\subsection{Water transport in the xylem}

We assume that the water flow in the xylem vessel consists of axial and radial components. It is generally accepted that the axial flow along the xylem is governed by Poiseuille's law (Frensch \& Steudle, 1989) such that the dimensional axial flux $\left(\mathrm{m}^{3} \mathrm{~s}^{-1}\right)$ is given by

$$
\hat{q}_{z}^{i}=-\hat{k}_{z}\left(\frac{d \hat{p}^{i}}{d \hat{z}}-\hat{\rho} \hat{g}\right), \quad \hat{k}_{z}=\sum_{j} \frac{\pi \hat{v}_{j}^{i} \hat{a}_{j}^{i 4}}{8 \hat{\mu}},
$$

where $\hat{p}^{i}$ is the internal fluid pressure $(\mathrm{Pa})$ in the xylem vessels, $\hat{\rho}$ is the density $\left(\mathrm{kg} \mathrm{m}^{-3}\right)$ of the sap, $\hat{g}$ is the gravitational acceleration $\left(\mathrm{m} \mathrm{s}^{-2}\right), \hat{k}_{z}$ is the xylem axial Poiseuille conductivity $\left(\mathrm{m}^{4} \mathrm{~Pa}^{-1} \mathrm{~s}^{-1}\right)$, and $\hat{\mu}$ is the viscosity (Pas). In general, $\hat{a}_{j}^{i}$ and $\hat{v}_{j}^{i}$ can vary with plant length, but aggregrate properties such as $\hat{k}_{z}$ are continuous. In Section 3 we will show that $\hat{k}_{z}$ varies by a small amount between plant zones, and therefore choose that $\hat{k}_{z}$ is constant across the plant. In addition, we assume that $\hat{\mu}$ and $\hat{\rho}$ are also constant. By conservation of mass, the axial flux along the xylem tubes is balanced by the radial flux into the tubes such that

$$
-\hat{k}_{z} \frac{d^{2} \hat{p}^{i}}{d \hat{z}^{2}}=\sum_{j} 2 \pi \hat{v}_{j}^{i} \hat{a}_{j}^{i} \hat{q}_{r}^{i},
$$


where $\hat{q}_{r}^{i}$ is the radial flux per unit area $\left(\mathrm{m} \mathrm{s}^{-1}\right)$ into the xylem vessels. In the root zone, water flows into the xylem from the soil, whereas in the leaf zone water flows out of the xylem into the mesophyll air-spaces which can then evaporate out to the air. The flow of water through the plant tissues is governed primarily by differences in pressure and hence mainly follows the passive apoplasmic pathway through the cortex tissue (Steudle \& Peterson, 1998). Following the approach of Steudle \& Peterson (1998), Molz (1981), and Landsberg \& Fowkes (1978) we treat the variety of tissues as a composite membrane and describe the radial flows $\left(\mathrm{ms}^{-1}\right)$ as

$$
\hat{q}_{r}^{R}=\hat{k}_{r}^{R}\left(\hat{p}^{\text {soil }}-\hat{p}^{R}\right), \quad \hat{q}_{r}^{L}=\hat{k}_{r}^{L}\left(\hat{p}^{L}-\hat{p}^{e x t}\right),
$$

where $\hat{q}_{r}^{R}$ is the radial flow in the root from soil to xylem, and $\hat{q}_{r}^{L}$ is the radial flow from the xylem to the leaf air-spaces. The tissue radial conductivities $\left(\mathrm{m} \mathrm{s}^{-1} \mathrm{~Pa}^{-1}\right)$ are $\hat{k}_{r}^{R}$ in the root, and $\hat{k}_{r}^{L}$ in the leaf, and $\hat{p}^{\text {soil }}(\mathrm{Pa})$ is the water pressure in the soil, and $\hat{p}^{e x t}(\mathrm{~Pa})$ is the pressure external to the xylem in the leaf air-spaces. Substituting the radial fluxes (3) into the water conservation equation (2) we obtain

$$
\begin{gathered}
-\hat{k}_{z} \frac{d^{2} \hat{p}^{L}}{d \hat{z}^{2}}=\hat{\alpha}^{L}\left(\hat{p}^{L}-\hat{p}^{e x t}\right), \\
-\hat{k}_{z} \frac{d^{2} \hat{p}^{S}}{d \hat{z}^{2}}=0, \\
-\hat{k}_{z} \frac{d^{2} \hat{p}^{R}}{d \hat{z}^{2}}=\hat{\alpha}^{R}\left(\hat{p}^{\text {soil }}-\hat{p}^{R}\right), \\
\text { where } \quad \hat{\alpha}^{L}=\sum_{j} 2 \pi \hat{v}_{j}^{L} \hat{a}_{j}^{L} \hat{k}_{r}^{L}, \quad \hat{\alpha}^{R}=\sum_{j} 2 \pi \hat{v}_{j}^{R} \hat{a}_{j}^{R} \hat{k}_{r}^{R},
\end{gathered}
$$

where $\hat{v}_{j}^{L}$ are the number of xylem vessels in the leaves, and $\hat{v}_{j}^{R}$ are the number of xylem vessels in the roots. Two boundary conditions are required to solve the model and we follow a similar approach to that of Landsberg \& Fowkes (1978). At the tip of the xylem in the root zone we prescribe zero flux of water as we assume that the xylem terminus in the roots is axially impermeable to water. At the xylem tip in the leaf zone we assume that the xylem vessel is open to the leaf air-spaces and prescribe the pressure to be $\hat{p}^{0}(\mathrm{~Pa})$. Since the architecture of the leaf air-spaces is inhomogeneous, it is possible that the pressure in the air-spaces can vary, and hence we also allow the possibility that $p^{0} \neq p^{e x t}$. The boundary conditions therefore are

$$
\hat{p}^{L}=\hat{p}^{0} \text { at } \hat{z}=0, \quad \frac{d \hat{p}^{R}}{d \hat{z}}-\hat{\rho} \hat{g}=0 \text { at } \hat{z}=\hat{z}_{3} .
$$

In addition we prescribe continuity of pressure and pressure flux at the zone boundaries, $\hat{z}_{1}$ and $\hat{z}_{2}$. Note that $\hat{k}_{z}$ is constant across the plant, resulting in the conditions of continuity of flux simplifying to continuity of pressure gradient. 


\begin{tabular}{|c|c|c|c|c|}
\hline Symbol & Description & Value & Units & Source \\
\hline $\bar{L} L, S, R$ & leaf, stem, root zones & $\begin{array}{c}- \\
\end{array}$ & 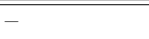 & $\begin{array}{c}- \\
\end{array}$ \\
\hline$i$ & zone index & - & - & - \\
\hline$j$ & xylem vessel index & - & - & - \\
\hline$\hat{v}$ & number of xylem vessels & Tables $4 \& 5$ & - & Percival (1921) \\
\hline$\hat{a}$ & xylem vessel radius & Tables 4 \& 5 & $\mathrm{~m}$ & Percival (1921) \\
\hline$\hat{z}$ & axial coordinate & variable & $\mathrm{m}$ & - \\
\hline$\hat{z}_{1}$ & position of leaf-stem boundary & 0.23 & $\mathrm{~m}$ & Kutschera et al. (2009) \\
\hline$\hat{z}_{2}$ & position of stem-root boundary & 0.33 & $\mathrm{~m}$ & Kutschera et al. (2009) \\
\hline$\hat{z}_{3}$ & length of plant & 0.81 & $\mathrm{~m}$ & Kutschera et al. (2009) \\
\hline$\hat{a}^{R}$ & radius of root & $2.48 \times 10^{-4}$ & $\mathrm{~m}$ & Jones et al. (1983) \\
\hline$\hat{a}^{L}$ & thickness of leaf & $1.38 \times 10^{-4}$ & $\mathrm{~m}$ & Araus et al. (1986) \\
\hline$\hat{n}$ & concentration of nutrient & variable & $\mathrm{mol} \mathrm{m}^{-3}$ & - \\
\hline$\hat{N}$ & concentration at $z=0$ & 0 & $\mathrm{~mol} \mathrm{~m}^{-3}$ & Section 2 \\
\hline$\hat{C}$ & typical concentration scale & variable & $\mathrm{mol} \mathrm{m}^{-3}$ & Section 3 \\
\hline$\hat{D}$ & diffusivity of nutrient in water & $10^{-9}$ & $\mathrm{~m}^{2} \mathrm{~s}^{-1}$ & Perry (1997) \\
\hline$\hat{F}$ & radial loading/unloading of nutrient & variable & $\mathrm{mol} \mathrm{m}^{-2} \mathrm{~s}^{-1}$ & - \\
\hline$\hat{f}$ & axial loading/unloading at $z=z_{3}$ & variable & $\mathrm{mol} \mathrm{m}^{-2} \mathrm{~s}^{-1}$ & - \\
\hline$\hat{k}_{z}$ & xylem axial conductivity & $2 \times 10^{-16}$ & $\mathrm{~m}^{4} \mathrm{~Pa}^{-1} \mathrm{~s}^{-1}$ & Section 3 \\
\hline$\hat{k}_{r}^{R}$ & root local radial conductivity & $1.2 \times 10^{-13}$ & $\mathrm{~m} \mathrm{~s}^{-1} \mathrm{~Pa}^{-1}$ & Jones 1983 \\
\hline$\hat{k}_{r}^{L}$ & leaf local radial conductivity & $1.35 \times 10^{-12}$ & $\mathrm{~m} \mathrm{~s}^{-1} \mathrm{~Pa}^{-1}$ & Section 3 \\
\hline$\hat{\alpha}^{L}$ & leaf effective radial conductivity & $3.87 \times 10^{-15}$ & $\mathrm{~m}^{2} \mathrm{~Pa}^{-1} \mathrm{~s}^{-1}$ & Section 3 \\
\hline$\hat{\alpha}^{R}$ & root effective radial conductivity & $1.11 \times 10^{-16}$ & $\mathrm{~m}^{2} \mathrm{~Pa}^{-1} \mathrm{~s}^{-1}$ & Section 3 \\
\hline$\hat{\mu}$ & fluid (water) viscosity in xylem & $8.9 \times 10^{-4}$ & $\mathrm{Pas}$ & standard \\
\hline$\hat{\rho}$ & fluid (water) density in xylem & $10^{3}$ & $\mathrm{~kg} \mathrm{~m}^{-3}$ & standard \\
\hline$\hat{g}$ & gravitational acceleration & 9.81 & $m s^{-2}$ & standard \\
\hline$\hat{q}_{z}$ & axial flux in xylem & variable & $m^{3} s^{-1}$ & - \\
\hline$\hat{q}_{r}$ & radial flux in xylem & variable & $m s^{-1}$ & - \\
\hline$\hat{p}$ & internal pressure in xylem & variable & $\mathrm{Pa}$ & - \\
\hline$\hat{p}^{\text {soil }}$ & soil pore water pressure & $-0.3 \times 10^{6}$ & $\mathrm{~Pa}$ & Campbell (2008) \\
\hline$\hat{p}^{e x t}$ & leaf-air-space pressure & $-1 \times 10^{6}$ & $\mathrm{~Pa}$ & Campbell (2008) \\
\hline$\hat{p}^{0}$ & xylem pressure at $z=0$ & $-1 \times 10^{6}$ & $\mathrm{~Pa}$ & Campbell (2008) \\
\hline$\hat{P}$ & typical pressure scale & $1 \times 10^{6}$ & $\mathrm{~Pa}$ & Section 3 \\
\hline
\end{tabular}

Table 1: Summary of dimensional symbols and their values.

\subsection{Nutrient transport in the xylem}

We assume that radial diffusion of the nutrients is fast and hence only consider their variation in the axial direction. Nutrients such as phosphate are loaded into the xylem radially at the root and unloaded in the stem and leaf regions. We follow the approaches of Thompson \& Holbrook (2003) and Jensen et al. (2011, 2012) and assume that the loading and unloading of nutrient into and out of the vessels, denoted by $\hat{F}^{i}\left(\mathrm{~mol} \mathrm{~m}^{-2} \mathrm{~s}^{-1}\right)$, is balanced by the flux of nutrient in the axial direction. However, unlike Thompson \& Holbrook (2003) and Jensen et al. (2011, 2012) we allow diffusion to contribute to the axial flux. Therefore conservation of $\hat{n}$ is given by

$$
\frac{d}{d \hat{z}}\left(\hat{n}^{i} \hat{q}_{z}^{i}-\pi \hat{v}_{j}^{i} \hat{a}_{j}^{i 2} \hat{D} \frac{d \hat{n}^{i}}{d \hat{z}}\right)=2 \pi \hat{v}_{j}^{i} \hat{a}_{j}^{i} \hat{F}^{i}
$$

where $\hat{D}$ is the diffusion coefficient $\left(\mathrm{m}^{2} \mathrm{~s}^{-1}\right)$ of phosphate in the xylem sap. Diffusion has previously been neglected since, in the main bulk of the fluid, 
the diffusive flux of nutrients is negligible compared to convection, such that the nutrient transport is mainly due to the flow of water. However, near the ends of the xylem vessel, diffusion can become important when fluid flow is slow or zero, for example at the xylem terminus in the root tip $\left(\hat{z}=\hat{z}_{3}\right)$. In addition, due to the zero fluid velocity at $\hat{z}=\hat{z}_{3}$, in a convection-only system, no boundary conditions can be applied to the system. For example, in a convection-only system the nutrient transport is governed by

$$
\frac{d}{d \hat{z}}\left(\hat{n}^{i} \hat{q}_{z}^{i}\right)=2 \pi \hat{v}_{j}^{i} \hat{a}_{j}^{i} \hat{F}^{i}
$$

and has the solution $\hat{n}^{i}=\frac{\int 2 \pi \hat{v}_{j}^{i} \hat{a}_{j}^{i} \hat{F}^{i} d \hat{z}+k}{\hat{q}_{z}^{i}}$ where $k$ is a constant. Since $\hat{q}_{z}^{i}=0$ at $\hat{z}=\hat{z}_{3}$, the only choice of $k$ that would keep $\hat{n}^{i}$ finite everywhere is $k=-\left.\int 2 \pi \hat{v}_{j}^{i} \hat{a}_{j}^{i} \hat{F}^{i} d \hat{z}\right|_{\hat{z}=\hat{z}_{3}}$. If another value of $k$ is chosen, the value of $\hat{n}^{i}$ would be infinite at $\hat{z}=\hat{z}_{3}$, which is unphysical. Applying continuity of $\hat{n}^{i}$ between the plant zones, results in $\hat{n}^{i}$ being fully determined for all $\hat{z}$ without having applied any external boundary conditions. It is unintuitive that a reduction in the order of the system from 2 for a combined convectiondiffusion system to 1 for a convection-only system results in the restriction that no boundary conditions can be applied, but the reason is solely due to the fact that $\hat{q}_{z}$ is prescribed to be equal to zero at $\hat{z}=\hat{z}_{3}$. At this point diffusion can no longer be neglected in comparison to convection, and we therefore include its effect and apply two boundary conditions to the system.

For generality we prescribe an axial flux of phosphate $\hat{f}\left(\mathrm{~mol} \mathrm{~m}^{-2} \mathrm{~s}^{-1}\right)$ into the xylem vessel at $\hat{z}=\hat{z}_{3}$, which is only possible if diffusion is included in the system. It is not known whether axial loading or unloading of phosphate occurs, but it is possible that axial flux can occur in response to the nutrient sink of the growing root tip. At the xylem terminus in the leaf we prescribe the concentration of phosphate $\hat{N}\left(\mathrm{~mol} \mathrm{~m}^{-3}\right)$, such that the boundary conditions are given by

$$
\begin{gathered}
\hat{n}^{L}=\hat{N} \text { at } \hat{z}=0, \\
-\pi \hat{v}_{j}^{R} \hat{a}_{j}^{R 2} \hat{D} \frac{d \hat{n}^{R}}{d \hat{z}}+\hat{q}_{z}^{R} \hat{n}^{R}=\pi \hat{v}_{j}^{R} \hat{a}_{j}^{R 2} \hat{f} \text { at } \hat{z}=\hat{z}_{3},
\end{gathered}
$$

where $\hat{q}_{z}^{R}=0$ at $\hat{z}=\hat{z}_{3}$. In addition, we prescribe continuity of phosphate concentration and phosphate flux at the zone boundaries $\hat{z}_{1}$ and $\hat{z}_{2}$. In Section 5 we choose that $\hat{N}=0$ to represent the low level of phosphate at $z=0$ due to its use in photosynthesis in the leaves, however for generality we retain $\hat{N}$ as a variable which is free to be chosen by the user.

\section{Nondimensionalisation and parameter values}

We nondimensionalise the equations by using the following scales $\hat{z}=\hat{z}_{3} z$, $\hat{p}=\hat{P} p, \hat{n}=\hat{C} n$, where we choose $\hat{P}$ to be equal to modulus of the pressure 
in the leaf air-space, $\hat{p}^{e x t}=-10^{6} \mathrm{~Pa}$ (Campbell, 2008), such that $\hat{P}=10^{6} \mathrm{~Pa}$. In addition we prescribe the soil pressure to equal $\hat{p}^{\text {soil }}=-0.3 \times 10^{6} \mathrm{~Pa}$ (Campbell, 2008). We leave the choice of $\hat{C}$, which is a typical concentration $\left(\mathrm{mol} \mathrm{m}^{-3}\right)$ of phosphate in the xylem vessels, to the end of this section. Note that only dimensional values are denoted with a hat (^) symbol. The nondimensional equations for water and nutrient transport are

$$
\begin{gathered}
\frac{d^{2} p^{L}}{d z^{2}}=M^{L}\left(p^{e x t}-p^{L}\right), \\
\frac{d^{2} p^{S}}{d z^{2}}=0, \\
\frac{d^{2} p^{R}}{d z^{2}}=M^{R}\left(p^{R}-p^{\text {soil }}\right), \\
\frac{d}{d z}\left(n^{i}\left(\frac{d p^{i}}{d z}-G\right)+\frac{1}{P e} \frac{d n^{i}}{d z}\right)=-F^{i},
\end{gathered}
$$

where the nondimensional parameters are defined in Table 2. $M^{L}$ is the ratio of leaf radial conductivity to axial conductivity, $M^{R}$ is the ratio of root radial conductivity to axial conductivity, $p^{e x t}$ is the nondimensional pressure in the leaf air-spaces, $p^{\text {soil }}$ is the nondimensional soil pore pressure, $G$ is the gravitational term, and $F^{i}$ is the nondimensional loading/unloading parameter. The nutrient Peclet number, $P e$, is the ratio of convective to diffusive transport and is given by $P e=\hat{k}_{z} \hat{P} / \pi \hat{v}_{j}^{i} \hat{a}_{j}^{i 2} \hat{D}$ which can, in general, vary across the plant. However, similarly as for $\hat{k}_{z}$, we expect $P e$ to be continuous and assume $P e$ is constant across the plant zones. The nondimensional boundary conditions are given by

$$
\begin{gathered}
p^{L}=p^{0} \text { at } z=0, \\
\frac{d p^{R}}{d z}-G=0 \text { at } z=1, \\
n^{L}=\phi \text { at } z=0, \\
\frac{1}{P e} \frac{d n^{R}}{d z}+\left(\frac{d p^{R}}{d z}-G\right) n^{R}=-f \text { at } z=1,
\end{gathered}
$$

where $p^{0}$ and $\phi$ are the nondimensional pressure and concentration at $z=0$, and $f$ is the axial loading/unloading parameter through the xylem tip in the root (see Table 2 for definitions and values). In addition we prescribe continuity of $p$, flux of $p, n$, and flux of $n$ at the nondimensional zone boundaries $z_{1}$ and $z_{2}$. We calculate the values of the nondimensional parameters based on typical values for a wheat plant and phosphate. Based on values measured for summer sowing wheat (Kutschera et al. (2009), pg 228) we consider the leaf-stem boundary to be at $\hat{z}_{1}=0.23 \mathrm{~m}$, the stem-root boundary to be at $\hat{z}_{2}=0.33 \mathrm{~m}$ and the length of the plant to be $\hat{z}_{3}=0.81 \mathrm{~m}$. We also assume that the xylem sap is similar in property to water such that the 
density is $\hat{\rho}=10^{3} \mathrm{~kg} \mathrm{~m}^{-3}$ and the viscosity is $\hat{\mu}=8.9 \times 10^{-4} \mathrm{~Pa}$ s. We use $\hat{g}=9.81 \mathrm{~m} \mathrm{~s}^{-2}$ for the gravitational acceleration and $\hat{D}=10^{-9} \mathrm{~m}^{2} \mathrm{~s}^{-1}$ for the diffusivity of phosphate in water (Perry, 1997).

\begin{tabular}{|c|c|c|}
\hline Symbol & Description & Value \\
\hline$z_{1}=\frac{\hat{z}_{1}}{\hat{z}_{3}}$ & nondimensional leaf-stem boundary & 0.3 \\
\hline$z_{2}=\frac{\ddot{z}_{2}}{\tilde{z}_{3}}$ & nondimensional stem-root boundary & 0.4 \\
\hline$M^{L}=\frac{\hat{\alpha}^{L} \hat{z}_{3}^{2}}{\hat{k}_{L}^{L}}$ & ratio of radial to axial conductivity in leaf & 13 \\
\hline$M^{R}=\frac{\hat{\alpha}^{R} \hat{z}_{3}^{2}}{\hat{k}_{z}^{R}}$ & ratio of radial to axial conductivity in root & 0.4 \\
\hline$G=\frac{\hat{\rho} \hat{g} \hat{z}_{3}}{\hat{P}}$ & ratio of gravity to pressure scale & 0.01 \\
\hline$P e=\frac{\hat{k}_{z} \hat{P}}{\pi \hat{v}_{j}^{i} \hat{a}_{j}^{i 2} \hat{D}}$ & ratio of convective to diffusive transport & $10^{7}$ \\
\hline$F^{L}=\frac{2 \pi \hat{v}_{j}^{L} \hat{a}_{j}^{L} \hat{z}_{3}^{2} \hat{F}^{L}}{\hat{k}_{z} \hat{P} \hat{C}}$ & ratio of leaf radial loading to axial transport & $-1^{*}$ \\
\hline$F^{S}=\frac{2 \pi \hat{v}_{j}^{S} \hat{a}_{j}^{S} \hat{z}_{3}^{2} \hat{F}^{S}}{\hat{k}_{z} \hat{P} \hat{C}}$ & ratio of stem radial loading to axial transport & $-0.5^{*}$ \\
\hline$F^{R}=\frac{2 \pi \hat{v}_{j}^{R} \hat{a}_{j}^{R} \hat{z}_{3}^{2} \hat{F}^{R}}{\hat{k}_{z} \hat{P} \hat{C}}$ & ratio of root radial loading to axial transport & $1^{*}$ \\
\hline$f=\frac{\pi \hat{v}_{j}^{R} \hat{a}_{j}^{R 2} \hat{z}_{3} \hat{f}}{\hat{k}_{z} \hat{P} \hat{C}}$ & ratio of axial loading to axial transport & $0.01^{*}$ \\
\hline$p^{0}=\frac{\hat{p}^{0}}{\hat{P}}$ & ratio of xylem pressure at $z=0$ to $\left|\hat{p}^{e x t}\right|$ & -1 \\
\hline$p^{\text {soil }}=\frac{\hat{p}^{\text {soil }}}{\hat{P}}$ & ratio of soil pressure to $\left|\hat{p}^{e x t}\right|$ & -0.3 \\
\hline$p^{e x t}=\frac{\hat{p}^{x x t}}{\hat{P}}$ & ratio of leaf-air-space pressure to $\left|\hat{p}^{e x t}\right|$ & -1 \\
\hline$\phi=\frac{\hat{N}}{\hat{C}}$ & ratio of phosphate concentration scales at $z=0$ & $0^{*}$ \\
\hline
\end{tabular}

Table 2: Summary of nondimensional symbols and typical values used based on those given in Table 1. The starred symbol $\left({ }^{*}\right)$ indicates that the value has been prescribed (see text in Section 3 for discussion).

Values for wheat root xylem radii and vessel numbers are given in Table 4 in Appendix A obtained from Percival (1921) (pg 39-40). Similarly, the values for wheat leaves are given in Table 5 in Appendix A from Percival (1921) (pg 57-59). Using the values in Table 4 and Table 5 we find that $\hat{k}_{z}$ is very similar in the two regions; in the roots $\hat{k}_{z}=1.93 \times 10^{-16} \mathrm{~m}^{4} \mathrm{~Pa}^{-1} \mathrm{~s}^{-1}$, and in the leaves $\hat{k}_{z}=2.01 \times 10^{-16} \mathrm{~m}^{4} \mathrm{~Pa}^{-1} \mathrm{~s}^{-1}$. Therefore we choose an average value of $\hat{k}_{z}=2 \times 10^{-16} \mathrm{~m}^{4} \mathrm{~Pa}^{-1} \mathrm{~s}^{-1}$. Similarly, we calculate that in the root zone $1 / P e=6.1 \times 10^{-7}$, and in the leaf zone $1 / P e=7.12 \times 10^{-8}$, such that $P e \approx 10^{7}$. This indicates that diffusion is negligible compared to convection in the main flow regime in all plant zones.

We use a wheat root radial conductivity of $\hat{k}_{r}^{R}=1.2 \times 10^{-13} \mathrm{~ms}^{-1} \mathrm{~Pa}^{-1}$ from Jones et al. (1983) based on a wheat root radius of $\hat{a}^{R}=2.48 \times$ $10^{-4} \mathrm{~m}$ and we estimate $\hat{k}_{r}^{L}$ by assuming that the leaf radial conductivity is proportional to the root radial conductivity through $\hat{k}_{r}^{L}=2 \pi \hat{a}^{R} k_{r}^{R} / \hat{a}^{L}$. Using a wheat leaf thickness of $\hat{a}^{L}=1.384 \times 10^{-4} \mathrm{~m}$ (Araus et al., 1986) we 
calculate $\hat{k}_{r}^{L}=1.35 \times 10^{-12} \mathrm{~ms}^{-1} \mathrm{~Pa}^{-1}$. Using Equation (7), we calculate $\hat{\alpha}^{R}=1.11 \times 10^{-16} \mathrm{~m}^{2} \mathrm{~s}^{-1} \mathrm{~Pa}^{-1}$ and $\hat{\alpha}^{L}=3.87 \times 10^{-15} \mathrm{~m}^{2} \mathrm{~s}^{-1} \mathrm{~Pa}^{-1}$. Finally we calculate $M^{R}=\hat{\alpha}^{R} \hat{z}_{3}^{2} / \hat{k}_{z} \approx 0.4, M^{L}=\hat{\alpha}^{L} \hat{z}_{3}^{2} / \hat{k}_{z} \approx 13$. With $\hat{P}=10^{6} \mathrm{~Pa}$, we calculate the gravitational term as $G=\hat{\rho} \hat{g} \hat{z}_{3} / \hat{P}=0.008 \approx 0.01$ and we see that the effect of gravity on water movement is small compared to the pressure gradient driven terms.

The values of the nondimensional radial $(F)$ and axial $(f)$ loading depend on knowledge of $\hat{F}$ and $\hat{f}$ which are, to the authors' knowledge, currently unknown in the literature. Considering the root zone we see that $F^{R} \approx$ $3 \times 10^{6} \frac{\hat{F^{R}}}{\hat{C}}, f \approx 18 \frac{\hat{f}}{\hat{C}}, \frac{F^{R}}{f}=1.7 \times 10^{5} \frac{\hat{F^{R}}}{\hat{f}}$, which indicates that if axial flux $\hat{f}$ did occur, when $\hat{f}$ is comparable to $\hat{F}^{R}$, its overall effect on the flow would be smaller than that of $\hat{F}^{R}$. Since $\hat{F}$ and $\hat{f}$ are unknown, we do not define a choice of $\hat{C}$ and, for this paper, assume that $F$ and $f$ are $O(1)$ and investigate the effect that varying $F$ and $f$ has on nutrient transport. We prescribe that $F^{R}$ is positive to represent loading of phosphate into the xylem vessels, and similarly prescribe that $F^{S}$ and $F^{L}$ are negative to represent unloading. Similarly, we prescribe that $f$ is positive, to represent axial flux of phosphate out of the xylem vessels in the root to be used for root growth.

In the following sections we solve for the water transport analytically and the nutrient transport analytically and numerically. In order to solve the nutrient transport equations analytically we consider the limit of high $P e$ and use the method of matched asymptotic expansions. We first determine the conditions for unidirectional water transport and investigate the effect that diffusion has on nutrient transport. We use the parameters values given in Table 2 unless otherwise stated.

\section{Results: Water transport}

Solving equations $(13)-(15)$ subject to the boundary conditions $(17)-(18)$ and continuity gives the following solution for the internal xylem pressure in the leaf, stem and root regions respectively;

$$
\begin{gathered}
p^{L}=A_{1} \sin \left(\sqrt{M^{L}} z\right)+A_{2} \cos \left(\sqrt{M^{L}} z\right)+p^{e x t}, \quad p^{S}=A_{3} z+A_{4}, \\
p^{R}=A_{5} e^{\sqrt{M^{R}} z}+A_{6} e^{-\sqrt{M^{R}} z}+p^{\text {soil }},
\end{gathered}
$$

where $A_{i}$ are constants determined from the boundary conditions and are given in the function 'constants' in the Maple file in the supplementary materials. For certain parameter values the flow can become multidirectional inside the xylem vessel, i.e. the velocity of the flow can change sign, which may be detrimental to nutrient transport from roots to the leaves. We determine the bounds on the parameter values that would give rise to 
multidirectional flow by solving $q_{z}^{L}=0, q_{z}^{S}=0$, and $q_{z}^{R}=0$, which indicates when the velocity in each zone is zero and hence may change sign.

In addition, we investigate the effect that having a difference between $p^{0}$ (the internal xylem pressure at $z=0$ ) and $p^{e x t}$ (the pressure external to the xylem in the leaf air-spaces) has on the flow. We will first consider the case of $p^{0}=p^{e x t}$ for $G=0$ and $G \neq 0$ and then the case of $p^{0} \neq p^{e x t}$ for $G=0$ and $G \neq 0$. In the following sections (4.1 and 4.2) we will classify the flow into 7 possible regimes depending on the parameter values; 4 are characterised by unidirectional flow (UDF), and 3 are characterised by multidirectional flow (MDF). These flows are summarised in Figure 11 and Table 3 in the Discussion (Section 6).

\subsection{Conditions for multidirectional flow where internal xylem pressure at $z=0$ equals leaf-air-space pressure $\left(p^{0}=p^{e x t}\right)$}

\subsubsection{Zero gravity $(G=0)$}

For $p^{0}=p^{e x t}$, if $G=0$, the conditions that satisfy $q_{z}^{R}=0$ and $q_{z}^{S}=0$ are

$$
p^{0}=p^{\text {soil }}, \quad M^{R}=0, \quad M^{L}=\frac{1}{4} \frac{\pi^{2}}{z_{1}^{2}},
$$

where $z_{1}$ is the nondimensional leaf length. If any of the conditions in Equation (22) are satisfied then $q_{z}^{R}=q_{z}^{S}=0$ for the entire length of the root and stem zones. The condition $q_{z}^{L}=0$ is satisfied for the same conditions except for the condition on $M^{L}$ where instead $M^{L}=\frac{1}{4} \frac{\pi^{2}}{z^{2}}$. This indicates that the position of where $q_{z}^{L}=0$ can vary and depends on $M^{L}$. Since the minimum of $M^{L}=\frac{1}{4} \frac{\pi^{2}}{z^{2}}$ is equal to $\frac{1}{4} \frac{\pi^{2}}{z_{1}^{2}}$, Equation (22) represents the critical value of $M^{L}$ for the entire length of the xylem vessel and we label this value of $M^{L}$ as $M_{c r i t}^{L}$; below this value the flow is unidirectional, whereas above this value the flow is multidirectional.

For a nondimensional leaf length of $z_{1}=0.3$ (corresponding to $\hat{z}_{1}=0.23$ m) $M_{c r i t}^{L}=27.4$, and Figure 2 shows the pressure, axial flux, and radial flow profiles for $M^{L}$ values above and below $M_{\text {crit }}^{L}$. For values of $M^{L}<M_{\text {crit }}^{L}$ (the $M^{L}=13$ line in Figure 2), the flow is positive UDF such that the axial flow is towards the leaves for all $z$. The radial flow in the leaf is negative, indicating outwards flow into the leaf air-spaces, and the radial flow in the root is positive, indicating inwards flow from the soil.

For values of $M^{L}>M_{\text {crit }}^{L}$ the flow is MDF, but its nature is dependent on the value of $M^{L}$. For a small increase above $M_{\text {crit }}^{L}$ (the $M^{L}=28$ line in Figure 2), the flow is divergent in the leaf zone. The point of divergence (the point where $q_{z}$ crosses 0 ) is near $z_{1}$, such that for the majority of the leaf zone the flow is towards $z=0$, and for the remainder of the leaf zone, and the stem and root zones, the flow is towards $z=1$. The radial flow in the leaf is out of the xylem vessel as previously, but the radial flow in the 


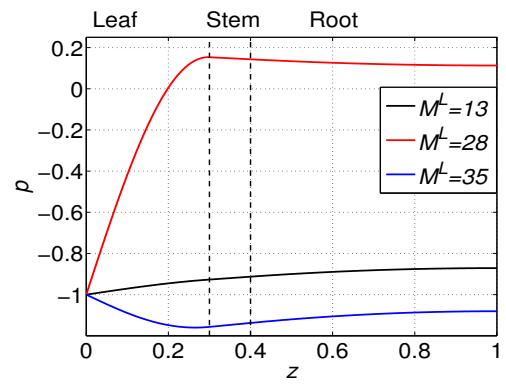

(a) pressure, $p$

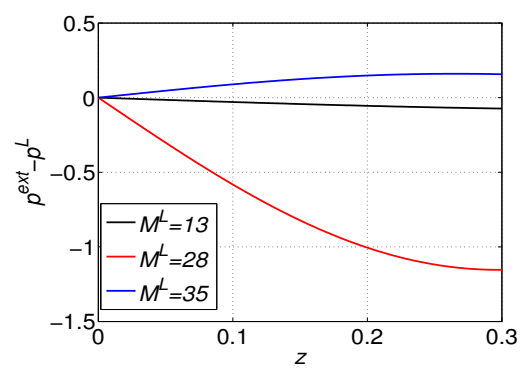

(c) radial flow in leaf, $p^{e x t}-p^{L}$

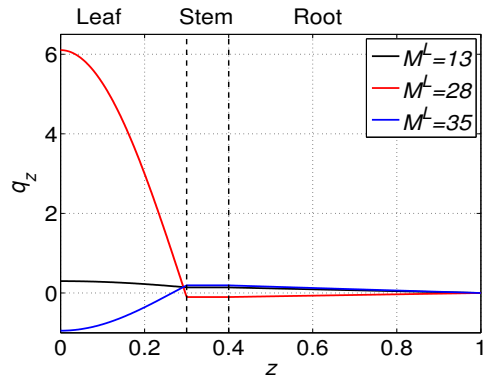

(b) axial flux, $q_{z}$

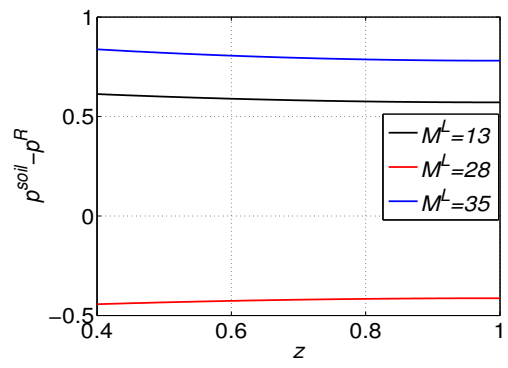

(d) radial flow in root, $p^{\text {soil }}-p^{R}$

Figure 2: Profiles of internal xylem pressure, axial flux, and radial flow against axial distance $z$ for $M^{L}$ values above and below the critical $M^{L}=27.4$, for $p^{\text {soil }}=-0.3, p^{0}=p^{\text {ext }}=-1, z_{1}=0.3, z_{2}=0.4$, $M^{R}=0.4, G=0$. The dashed lines represent the plant zone boundaries between leaf and stem, and stem and root. Positive radial flow indicates inwards flow into the xylem, whereas negative radial flow indicates outwards flow out of the xylem.

root is negative indicating outwards flow from the xylem vessel to the soil. For a large increase above $M_{\text {crit }}^{L}$ (the $M^{L}=35$ line in Figure 2) the flow is convergent in the leaf zone, with inwards radial flow in both the leaf and root zones, such that flow is driven down from the xylem tip in the leaf as well as up from the roots. Both the divergent and convergent leaf MDFs seem unphysical, and therefore $M_{c r i t}^{L}$ possibly represents the maximum value of $M^{L}$ that a plant can have. The critical value of $M^{L}$ is given by

$$
M^{L} \equiv \frac{\hat{\alpha}^{L} \hat{z}_{3}^{2}}{\hat{k}_{z}}=\frac{1}{4} \frac{\pi^{2}}{z_{1}^{2}}
$$

and therefore suggests a critical relationship between the ratio of radial to axial conductivity and the ratio of leaf length to plant length. For $p^{0}>p^{\text {soil }}$ the flow is negative (towards to the roots) UDF, with inwards radial flow in the leaves and outwards radial flow in the roots, and hence the complete opposite of positive UDF. For this research however, we will focus mainly on the physical case of $p^{0}, p^{e x t}<p^{\text {soil }}$ and $M^{L}<M_{\text {crit }}^{L}$. 


\subsubsection{Non-zero gravity $(G \neq 0)$}

For $G=0$, the critical parameter values that give rise to MDF are decoupled as shown in Equation 22. Conversely when $G \neq 0$, the parameters couple together, such that the position at which zero flux occurs (and hence gives rise to MDF) is a function of $G, M^{L}, M^{R}, z_{1}, z_{2}, p^{0}$, and $p^{\text {soil }}$. We rewrite this critical function in terms of $p_{c r i t}^{0}$ which represents the critical value of $p^{0}$ that would give rise to MDF for $0 \leq z \leq 1$ and Figure 3 shows the effect of $G$ on $p_{c r i t}^{0}$ for $M^{L}=13\left(<M_{c r i t}^{L}\right)$. The inclusion of $G$ causes $p_{\text {crit }}^{0}$ to vary with $z$ and decrease below $p^{\text {soil }}$ (the $G=0$ line) in all zones except in the leaf zone. The variation of $p_{\text {crit }}^{0}$ leads to 5 possible flow regimes:

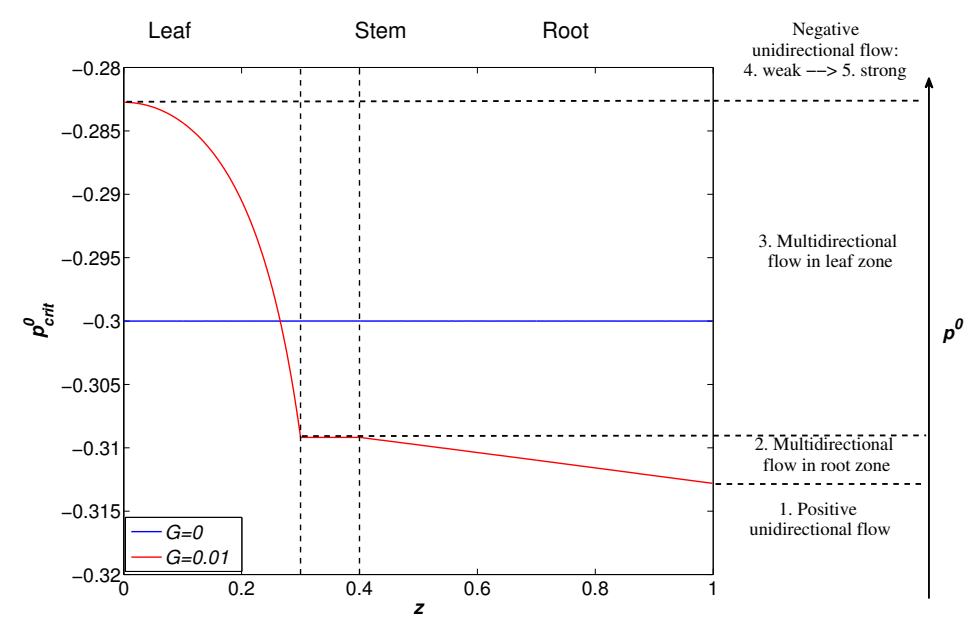

Figure 3: Effect of gravity on the critical value of $p^{0}$ that would give rise to multidirectional flow, $p_{\text {crit }}^{0}$, against axial distance $z$ for $M^{L}=13$ for $p^{\text {soil }}=-0.3, p^{0}=p^{\text {ext }}, z_{1}=0.3, z_{2}=0.4, M^{R}=0.4$. There are five possible flow regimes depending on the value of $p^{0}$.

1. positive UDF for $p^{0}<\left.p_{c r i t}^{0 R}\right|_{z=1}$,

2. divergent MDF in the root zone for $\left.p_{\text {crit }}^{0 R}\right|_{z=1}<p^{0}<p_{\text {crit }}^{0 S}$,

3. divergent MDF in the leaf zone for $p_{c r i t}^{0 S}<p^{0}<\left.p_{\text {crit }}^{0 L}\right|_{z=0}$,

4. weak negative UDF for $\left.p_{\text {crit }}^{0 L}\right|_{z=0}<p^{0}<p^{\text {soil }}-\frac{2 G e^{M^{R}\left(1+z_{2}\right)}}{\sqrt{M^{R}} \kappa_{1}}$,

5. strong negative UDF for $p^{0}>p^{\text {soil }}-\frac{2 G e^{M^{R}}\left(1+z_{2}\right)}{\sqrt{M^{R}} \kappa_{1}}$,

which are shown in Figure 4.

The different flow regimes occur as the difference between $p^{0}$ and $p^{\text {soil }}$, which is the primary cause of flow, decreases. The flow regimes (1), (5), and (3) are equivalent to the positive UDF, negative UDF, and divergent 
leaf MDF regimes respectively, discussed in Section 4.1.1. The inclusion of gravity therefore leads to additional flow regimes (2) and (4), radial flow profiles of which are shown in Figure 5. MDF in the root zone is characterised by outwards radial flow in the leaves, but with both inwards and outwards radial flow in the root zone.

The remaining regime, weak negative UDF, is characterised by outwards radial flow in both the leaf and root zones. The transition from weak to strong negative UDF occurs when the gradient of $q_{z}^{L}$ switches from negative to positive, which causes the switch from outwards to inwards leaf radial flow. However, for the parameter values of interest, this transition occurs for $p^{0}>p^{\text {soil }}$, and hence outside the regime of interest. Note that MDF cannot occur in the stem because we prescribe that radial flow occurs only in the root and leaf zones.

For the case $G=0$, if $p^{0}=p^{\text {soil }}$ there would be no flow for all $z$. With the inclusion of gravity, if $p^{0}=p^{\text {soil }}$, flow occurs but its nature is dependent on $p_{c r i t}^{0}$. For example for $M^{L}=13,\left.p_{c r i t}^{0}\right|_{z=0}$ is greater than $p^{\text {soil }}$, therefore for $p^{0}=p^{\text {soil }}$, MDF in the leaf zone will occur. However, if $M^{L}$ is smaller, for example $M^{L}=3$ as in Figure 6, then negative UDF can occur. In fact, it can be seen that if even if $p^{0}<p^{\text {soil }}$ negative UDF will occur. The consequence of this is that even a seemingly preferential pressure difference $\left(p^{0}\right.$ less than $\left.p^{\text {soil }}\right)$ for positive flow can lead to negative flow from leaves to roots. To ensure that only positive UDF occurs, $p^{0}$ should be below the minimum $p_{c r i t}^{0}$ for all regions, which is (for the parameter values of interest) in the root region at $z=1$ and is given by

$$
\begin{aligned}
& p_{c r i t \mid z=1}^{0 R}=p^{s o i l}+ \\
& \frac{G e^{-\sqrt{M^{R}}}\left(z_{2}+1\right)}{2}\left(\frac{\kappa_{1}}{\sqrt{M^{R}}}+\kappa_{2}\left(z_{1}-z_{2}\right)-\frac{\kappa_{2} \tan \left(\sqrt{M^{L}} z_{1}\right)}{\sqrt{M^{L}}}\right),
\end{aligned}
$$

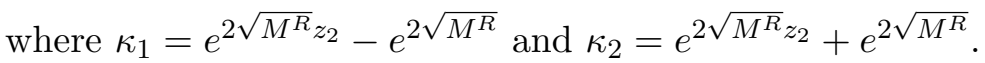

\subsection{Conditions for multidirectional flow where internal xylem pressure and leaf air-space pressure differ $\left(p^{0} \neq p^{e x t}\right)$}

\subsubsection{Zero gravity $(G=0)$}

For the case where the internal xylem pressure at $z=0$ and leaf air-space pressure differ $\left(p^{0} \neq p^{e x t}\right)$, the critical values which give rise to multidirectional flow of $M^{L}$ and $p^{0}$ again couple. If $G=0, q_{z}^{R}=q_{z}^{S}=0$ if

$$
M^{R}=0, \quad p^{0}=\cos \left(\sqrt{M^{L}} z_{1}\right)\left(p^{\text {soil }}-p^{e x t}\right)+p^{e x t} .
$$

At $z=z_{1}, q_{z}^{L}=0$ is satisfied by the same conditions, therefore Equation (25) is valid only for $z_{1} \leq z \leq 1$ (the stem and the root zones only) and we 


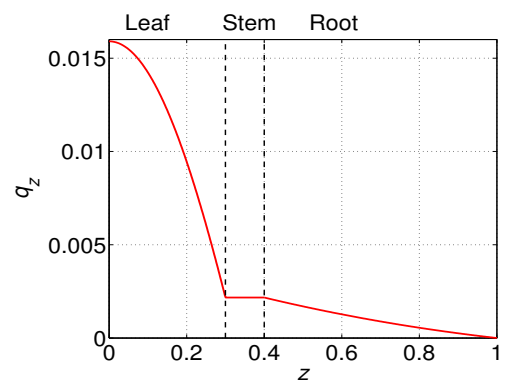

(a) positive UDF $\left(p^{0}=-0.32\right)$

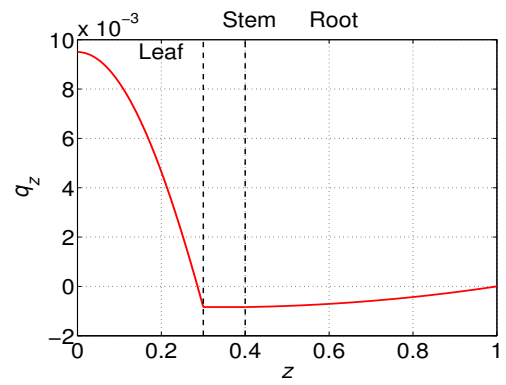

(c) MDF in the leaf $\left(p^{0}=-0.305\right)$

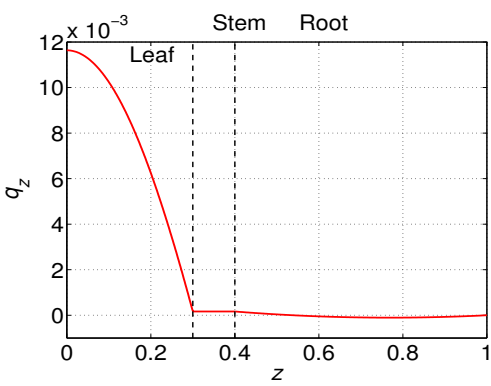

(b) $\mathrm{MDF}$ in the root $\left(p^{0}=-0.31\right)$

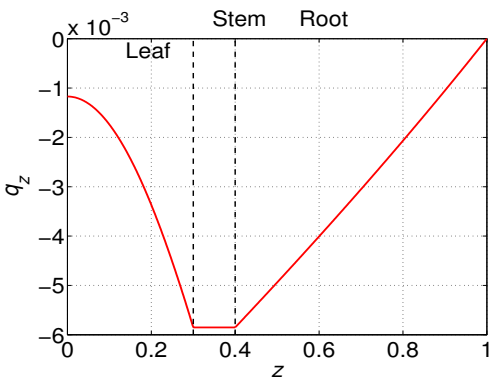

(d) weak negative UDF $\left(p^{0}=-0.28\right)$

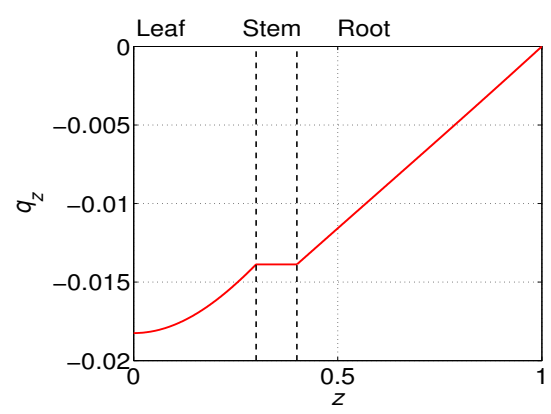

(e) strong negative UDF ( $\left.p^{0}=-0.24\right)$

Figure 4: Profiles of xylem flow velocity profiles against axial distance $z$ for increasing $p^{0}$, for $M^{L}=13, M^{R}=0.4, p^{\text {soil }}=-0.3, p^{0}=p^{\text {ext }}$, $z_{1}=0.3, z_{2}=0.4, G=0.01$. UDF stands for unidirectional flow, MDF stands for multidirectional flow. The conditions for the different flow regimes are given in Table 3. The dashed lines represent the plant zone boundaries between leaf and stem, and stem and root. 


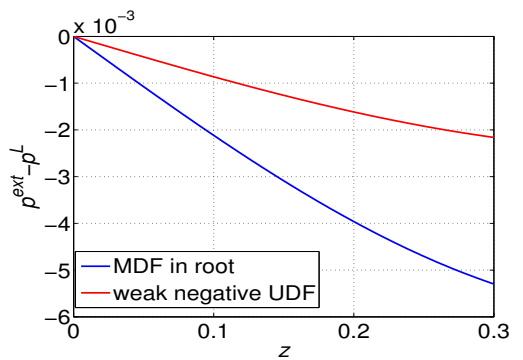

(a) radial flow in leaf

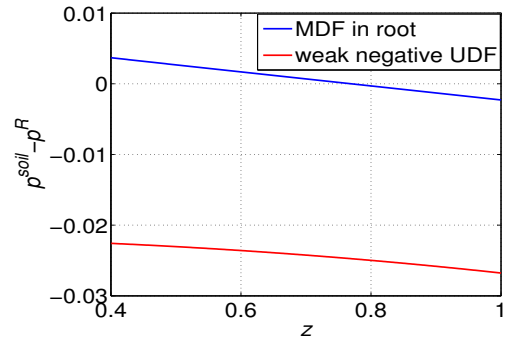

(b) radial flow in root

Figure 5: Radial flow profiles in leaf and roots for the cases of MDF in root $\left(p^{0}=-0.31\right)$, and weak negative UDF $\left(p^{0}=-0.28\right)$ for $M^{L}=13$, $M^{R}=0.4, p^{\text {soil }}=-0.3, p^{0}=p^{\text {ext }}, z_{1}=0.3, z_{2}=0.4, G=0.01$.

Positive radial flow indicates inwards flow into the xylem, whereas negative radial flow indicates outwards radial flow.

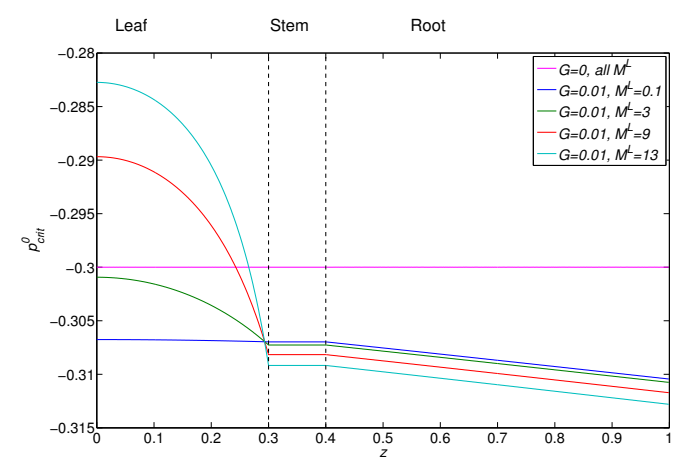

Figure 6: Effect of gravity on the critical value of $p^{0}$ that would give rise to multidirectional flow, $p_{\text {crit }}^{0}$, against axial distance $z$ for varying $M^{L}$, $p^{\text {soil }}=-0.3, p^{0}=p^{\text {ext }}, z_{1}=0.3, z_{2}=0.4, M^{R}=0.4$. The dashed lines represent the plant zone boundaries between leaf and stem, and stem and root.

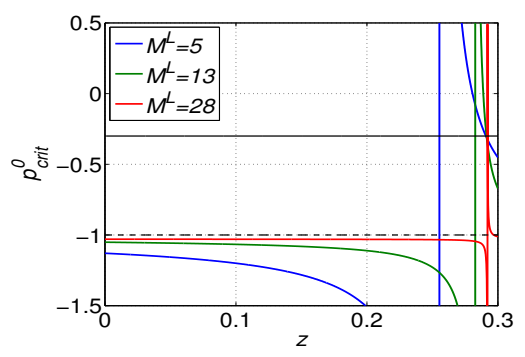

(a) large $M^{L}$

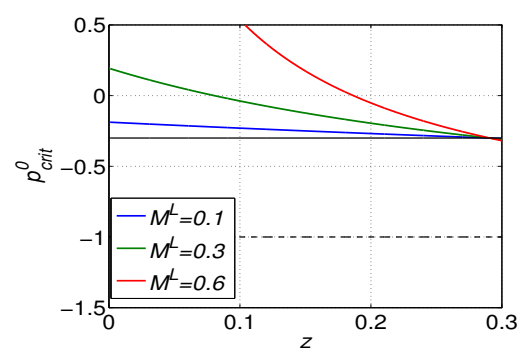

(b) small $M^{L}$

Figure 7: Variation of critical $p^{0}$ in the leaf region against axial distance $z$ for increasing $M^{L}$ for $p^{\text {soil }}=-0.3$, $p^{\text {ext }}=-1, z_{1}=0.3, z_{2}=0.4$, $M^{R}=0.4, G=0$. The solid black line represents $p^{\text {soil }}$ and the dashed black line represents $p^{\text {ext }}$. 
therefore label this critical value of $p^{0}$ as $p_{\text {crit }}^{0, S, R}$. For the parameter values of interest, increasing $M^{L}$ increases the magnitude of $p_{\text {crit }}^{0, S, R}$, and for values of $p^{0}<p_{\text {crit }}^{0, S, R}$ positive UDF occurs in the stem and root zone, whereas for $p^{0}>p_{\text {crit }}^{0, S, R} \mathrm{MDF}$ would result.

For $z \leq z_{1}$ (the leaf zone), the critical $p^{0}$ is a function of $z$, and its behaviour depends strongly on the value of $M^{L}$;

$$
\begin{aligned}
& p_{\text {crit }}^{0}=p^{e x t}+\frac{\sqrt{M^{R}}\left(p^{e x t}-p^{s o i l}\right) \kappa_{1} \cos \left(\sqrt{M^{L}} z\right)}{f_{1}(z)+f_{2}(z)}, \\
& f_{1}(z)=-\sqrt{M^{R}} \kappa_{1} \cos \left(\sqrt{M^{L}}\left(z-z_{1}\right)\right), \\
& f_{2}(z)=\sqrt{M^{L}}\left(\kappa_{2}+\sqrt{M^{R}}\left(z_{1}-z_{2}\right) \kappa_{1}\right) \sin \left(\sqrt{M^{L}}\left(z-z_{1}\right)\right) .
\end{aligned}
$$

Note that at $z=z_{1}$, Equation (26) is equivalent to Equation (25). Figure 7 shows the variation of the critical values of $p^{0}$ with $z$ in the leaf region for increasing values of $M^{L}$. Note that the solid black line represents $p^{\text {soil }}$ and the dashed black line represents $p^{e x t}$. The behaviour of $p_{\text {crit }}^{0}$ varies significantly for large and small $M^{L}$ and we can see that for large $M^{L}$ as in Figure 7(a), the solution displays discontinuous behaviour such that there is an inflection point within $0 \leq z \leq z_{1}$.

Only for the range $\left.p_{c r i t}^{0}\right|_{z=0}<p^{0}<\left.p_{c r i t}^{0}\right|_{z=z_{1}}$, does positive UDF exist. Outside of this range, MDF occurs: to the right of the inflection point, values of $p^{0}$ above $p_{c r i t}^{0}$ gives rise to divergent MDF, whereas to the left of the inflection point values of $p^{0}$ below $p_{c r i t}^{0}$ gives rise to convergent MDF.

The nature of the positive UDF that occurs depends on whether $p^{0}$ is smaller or larger than $p^{\text {ext }}$. For values of $\left.p_{\text {crit }}^{0}\right|_{z=0}<p^{0}<p^{e x t}$ weak positive UDF flow occurs which is characterised by some or total inwards radial flow in the leaf zone (as opposed to total outwards leaf radial flow for positive UDF seen in Section 4.1). This is simply because for this range there is enough of a pressure difference between $p^{0}$ and $p^{e x t}$ to cause a flow between them.

Increasing $M^{L}$ decreases the range of allowable $p^{0}$, and for values of $M^{L}>M_{\text {crit }}^{L}$ (the $M^{L}=28$ line on Figure $7(\mathrm{a})$ ), the range of allowable $p^{0}$ decreases below $p^{\text {ext }}$, in fact for $M^{L}=M_{\text {crit }}^{L},\left.p_{\text {crit }}^{0}\right|_{z=1}=p^{\text {ext }}$ as expected from Equation (25).

For very small values of $M^{L}$ the inflection point of $p_{\text {crit }}^{0}$ does not occur, as can be seen in Figure 7(b). For this case, positive UDF is obtained for $p^{0}<\left.p_{\text {crit }}^{0}\right|_{z=1}$, divergent leaf MDF for $\left.p_{\text {crit }}^{0}\right|_{z=0}<p^{0}<\left.p_{c r i t}^{0}\right|_{z=z_{1}}$, and weak negative UDF for $p^{0}>\left.p_{c r i t}^{0}\right|_{z=0}$.

\subsubsection{Non-zero gravity $G \neq 0$}

The addition of $G$ increases the complexity of the solution for the critical parameter values, however, conversely to the case of $p^{0}=p^{e x t}$, the effect of 
varying $G$ has only a small effect on the magnitude of $p_{c r i t}^{0}$ due to the small value of $G$ and large variation of $p_{c r i t}^{0}$. Similarly as for the case of $p^{0}=p^{e x t}$, the inclusion of $G$ reduces $p_{\text {crit }}^{0}$ below the $G=0$ case in the stem and root regions, and causes a variation of $p_{\text {crit }}^{0}$ in the root region with $z$ such that $\left.p_{c r i t}^{0}\right|_{z=1}$ is less than that at $\left.p_{c r i t}^{0}\right|_{z=z_{1}}$. The profile of $p_{c r i t}^{0}$ in the leaf region displays the same discontinuous behaviour as for the case of $G=0$ and the inclusion of $G$ also causes a very slight reduction in $p_{c r i t}^{0}$. The variation of $p_{\text {crit }}^{0}$ with $z$ in the root region introduces another possible flow regime such that there are 5 (compared to 4 for $G=0$ and high $M^{L}$ )

1. convergent MDF in the leaf zone $p^{0}<\left.p_{\text {crit }}^{0}\right|_{z=0}$,

2. positive weak UDF $\left.p_{\text {crit }}^{0}\right|_{z=0}<p^{0}<p^{e x t}$,

3. positive UDF $p^{e x t}<p^{0}<\left.p_{c r i t}^{0}\right|_{z=1}$,

4. divergent $\mathrm{MDF}$ in the root zone $\left.p_{c r i t}^{0}\right|_{z=1}<p^{0}<\left.p_{c r i t}^{0}\right|_{z=z_{1}}$,

5. divergent MDF in the leaf zone $p^{0}>\left.p_{\text {crit }}^{0}\right|_{z=z_{1}}$.

Therefore to ensure UDF, $p^{0}$ should lie in the range between $\left.p^{0 L}\right|_{z=0}$ and $\left.p^{0 R}\right|_{z=1}$ which are given in Appendix B.

\section{Results: Nutrient Transport}

We now consider the transport of phosphate in the xylem vessels. The following model is valid for all flow regimes (multidirectional and unidirectional), but we will only present results for the case of positive UDF which is the optimal flow regime for nutrient transport from the roots to the leaves. We assume that the flux of phosphate is due to both convection and diffusion and investigate the effect a phosphate sink in the roots has on transport. We solve the nutrient transport analytically in the limit of large $P e$ and numerically for all $P e$. The numerical solution was obtained using the bvp4c solver in Matlab to solve equations (13)-(16) with the boundary conditions (17) to (20) and continuity at the zone boundaries.

We now describe the analytical procedure. For sufficiently large $P e$ we consider the limit that $\epsilon=1 / P e \rightarrow 0$. However, the result is a singular equation for nutrient transport since the highest derivative is multiplied by $\epsilon$. To regularise the system, we assume that the flow in each zone can be split into one central bulk region and two boundary regions. In the bulk region convection dominates, but in the boundaries both convection and diffusion are important as the solution rapidly adjusts to the boundary conditions (for an introduction on boundary layer methods see Hinch (1991)). Diffusion is especially important when convection is small as the flow velocity tends to zero at the xylem terminus in the root $(z=1)$. We seek boundary layer solutions near $z=0$ and $z=1$ to satisfy the boundary conditions (19) and 
(20), and also at the zone boundaries $z_{1}$ and $z_{2}$ to satisfy the continuity boundary conditions. We neglect the effect of gravity as its effect is small, and as an example assume that $F$ is constant.

We find that the general solution to Equations (13) to (16) exhibit boundary layers near $z=0$ in the leaf, $z=z_{1}$ in the stem and at $z=z_{2}$ and $z=1$ in the root. Hence, when we impose the continuity conditions at leading order, we find that there are no rapid variations in the leaf at $z=z_{1}$ which we require to balance the rapidly varying concentration in the stem boundary layer. The result is that the coefficient of the boundary layer terms in the stem is zero to leading order. Similarly, we find that the coefficient of the boundary layer term at $z=z_{2}$ in the root must also be zero to leading order. Therefore, we neglect the boundary layers between the different zones as they are, at largest, of order $P e^{-\frac{1}{2}}$ and do not contribute to the problem as significantly as those at $z=0$ and $z=1$.

The full analytical method is given in Appendix C, where the leading order composite solution of $n$ is given by

$$
\begin{aligned}
& n^{L}=\frac{-F^{L} z+k_{4}}{\frac{d p^{L}}{d z}}+\left(\phi-\frac{k_{4}}{\tilde{L}_{1}}\right) e^{-\tilde{L}_{1} P e z}+O\left(P e^{-\frac{1}{2}}\right), \\
& n^{S}=\frac{-F^{S} z+k_{2}}{A_{3}}+O\left(P e^{-\frac{1}{2}}\right), \\
& n^{R}=\frac{F^{R}(1-z)}{\frac{d p^{R}}{d z}}+ \\
& f \sqrt{P e} \frac{1}{2} \sqrt{\frac{2 \pi}{\tilde{R}_{11}}} e^{\frac{1}{2}} \tilde{R}_{11} P e(1-z)^{2}\left(\operatorname{erf}\left(\frac{1}{2} \sqrt{2 \tilde{R}_{11} P e}(1-z)\right)-1\right)+O\left(P e^{-\frac{1}{2}}\right),
\end{aligned}
$$

where $k_{2}, k_{4}, \tilde{L}_{1}$, and $\tilde{R}_{11}$ are constants given in Equations (C.40), (C.31), and (C.10) respectively in Appendix C. We can see in Equation (29) that the boundary layer solution near $z=1$ is entirely due to $f$ which is the axial flux of nutrient through the xylem tip. If $f=0$, there would be no boundary layer at the root tip and the convection-only solution would be applicable. However, we can see that even for small $f$, the root tip boundary layer solution may be important as it is multiplied by $\sqrt{P e}$. Expressing the boundary layer solution at the root tip in terms of erfc (where $\operatorname{erfc}(x)=$ $1-\operatorname{erf}(x))$ we find that

$$
n^{B L}=-f \sqrt{P e} \frac{1}{2} \sqrt{\frac{2 \pi}{\tilde{R}_{11}}} e^{\frac{1}{2} \tilde{R}_{11} P e(1-z)^{2}} \operatorname{erfc}\left(\frac{1}{2} \sqrt{2 \tilde{R}_{11} P e}(1-z)\right) .
$$

For high $P e$, the limit of Equation (30) as $P e$ increases is $-\infty$, which is unphysical. We therefore surmise that if a flux $f$ occurs, it must be, at largest, of the order $P e^{-\frac{1}{2}}$ to result in an $O(1)$ solution at the root tip. 
Figure 8 shows the profile of the analytical and numerical solutions of $n$ against $z$ for increasing $P e$. We can see that as $P e$ increases there is good agreement between the analytical and numerical solutions except near the zone boundaries where we have neglected the boundary layers between zones since they are of order $P e^{-\frac{1}{2}}$. The estimated $P e$ in the xylem vessels is $P e \approx 10^{7}$, but we can see that even for $P e=2000$ (Figure $8(\mathrm{~b})$ ), the analytical and numerical solutions agree closely, and the zone boundaries are not visible, therefore the asymptotic solution is certainly valid for $P e>$ 2000. Increasing $P e$ also changes the solution in the root region due to the dependence of the boundary layer solution on $P e$, this implies that the significance of an axial flux $f$ increases as $P e$ increases. This is unlike the convection-only case, or for the case $f=0$, where for increasing $P e$, the solution tends to a constant solution. However, note that (as discussed in the previously), we expect that the magnitude of $f$ should be limited to be at largest $O\left(P e^{-\frac{1}{2}}\right)$ so as to result in only non-infinite values of $n$.

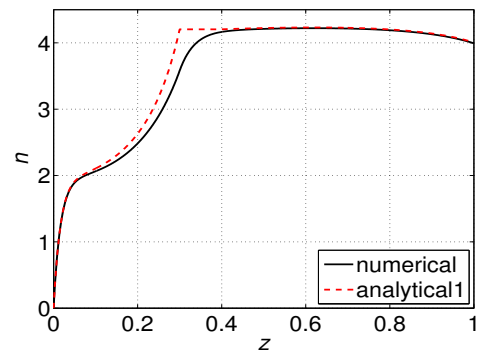

(a) $P e=200$

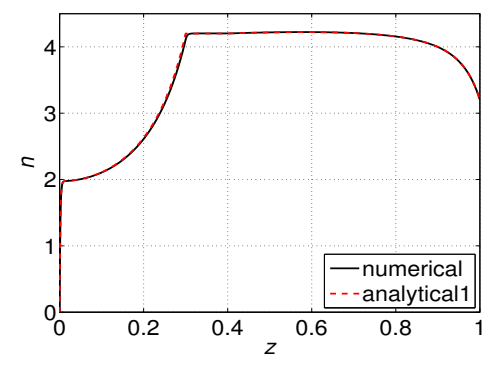

(b) $P e=2000$

Figure 8: Profiles of $n$ against $z$ for comparing analytical and numerical solutions for increasing Pe, $p^{\text {soil }}=-0.3, p^{0}=p^{\text {ext }}=-1, G=0$, $f=0.01, F=(0,0,1), \phi=0, z_{1}=0.3, z_{2}=0.4, M^{R}=0.4, M^{L}=13$.

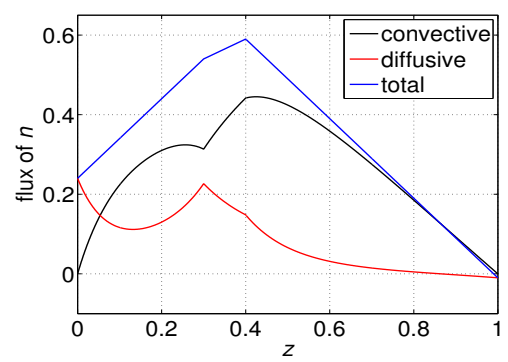

(a) $P e=50$

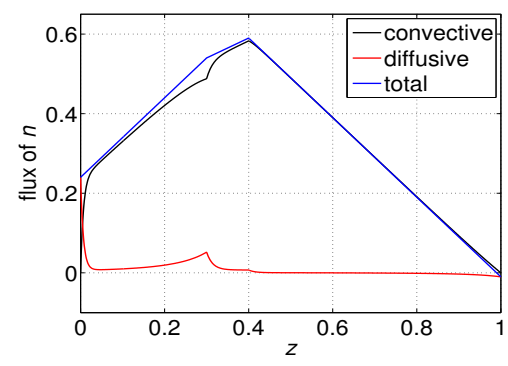

(b) $P e=500$

Figure 9: Components of nutrient flux against $z$, for $F=(-1,-0.5,1)$,

$$
\begin{gathered}
p^{\text {soil }}=-0.3, p^{0}=p^{\text {ext }}=-1, G=0.01, f=0.01, \phi=0, z_{1}=0.3, \\
z_{2}=0.4, M^{R}=0.4, M^{L}=13 .
\end{gathered}
$$


Figure 9 displays the profiles of the diffusive and convective flux components (numerical solution) against $z$ for $P e=50$ and $P e=500$. We can see that the convective flux is the dominant component of flux except near the ends of the xylem vessel ( $z=0$ and $z=1$ ) where the convective flux falls to zero and the diffusive flux becomes large. The diffusive terms are responsible for adjusting the convection-only solution to the boundary conditions at the ends of the xylem vessel. In particular, we can see that the diffusive flux undergoes a rapid change near $z=0$, and a gradual change near $z=1$. Additional contributions of the diffusive flux can also be seen at the zone boundaries $\left(z_{1}=0.3\right.$ and $\left.z_{2}=0.4\right)$, as the solution changes zone. Note that the numerical solution includes flux continuity at the zone boundaries for all orders of $P e$. Figure 10 shows the profiles of $n$ against $z$ for decreasing values

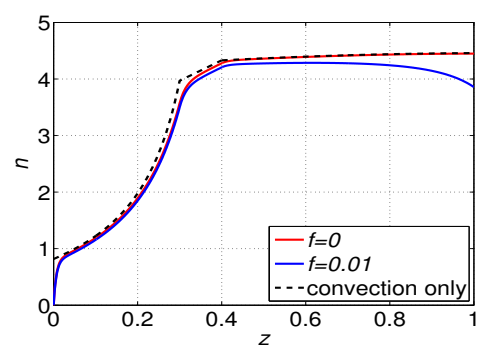

(a) $p^{e x t}=-1$

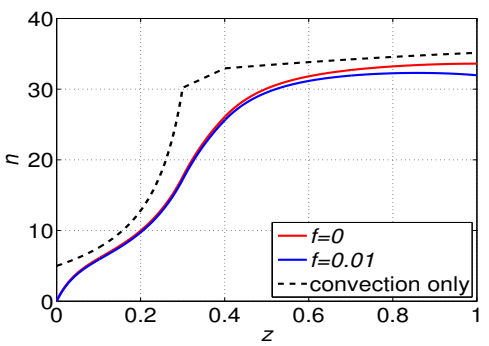

(c) $p^{e x t}=-0.4$

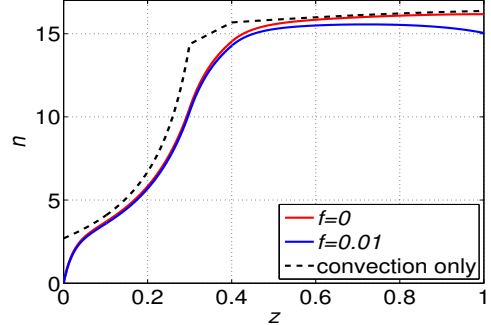

(b) $p^{e x t}=-0.5$

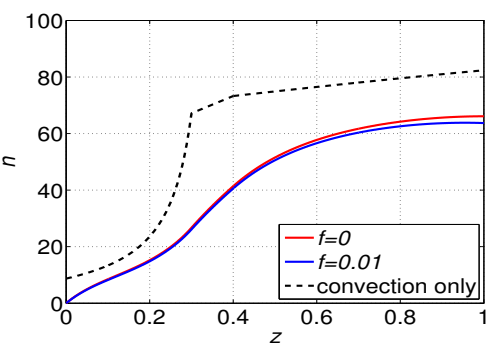

(d) $p^{e x t}=-0.35$

Figure 10: Profiles of $n$ against $z$ for increasing $p^{\text {ext }}$ compared to the convection only solution, for $F=(-1,-0.5,1), p^{\text {soil }}=-0.3, p^{0}=p^{\text {ext }}$, $G=0.01, \phi=0, z_{1}=0.3, z_{2}=0.4, M^{R}=0.4, M^{L}=13$.

of $p^{e x t}$ and for increasing $f$ compared to the convection-only solution. For high $P e$ the solution for $f=0$ should be very similar to the convection-only solution. However this is only the case when the difference between $p^{e x t}$ and $p^{\text {soil }}$ is large, giving rise to high water flow velocities. During the night, or at times of increased humidity, $p^{e x t}$ increases, decreasing the difference between $p^{e x t}$ and $p^{\text {soil }}$ and hence causing slower water flow velocities. The slower the water flow, the greater the contribution that diffusion makes to nutrient transport, as can be seen in Figure 10(d) where $p^{e x t}=-0.35$ and both the 
$f=0$ and $f=0.01$ solution of $n$ largely deviates from the convection-only solution. As $p^{e x t}$ increases (becomes less negative) the magnitude of $n$ increases within the xylem vessels indicating build up, but this is because a constant value of $F$ has been prescribed in each of the plant zones. The functional form of $F$ is generally unknown, but more-complicated functions, such as concentration or $z$ dependent, can be easily be input into the model.

\section{Discussion}

We have presented a model for the transport of water and nutrients within the xylem vessels of a wheat plant. We assumed that the flow of water is due to evapotranspiration which creates a negative potential at the leaves that drives water upwards from the roots. Depending on the parameter values, we have seen that the flow can be classified into 7 different regimes; 4 unidirectional flows (UDF) and 3 multidirectional flows (MDF), which are shown in Figure 11. We have considered the 2 separate cases of pressure

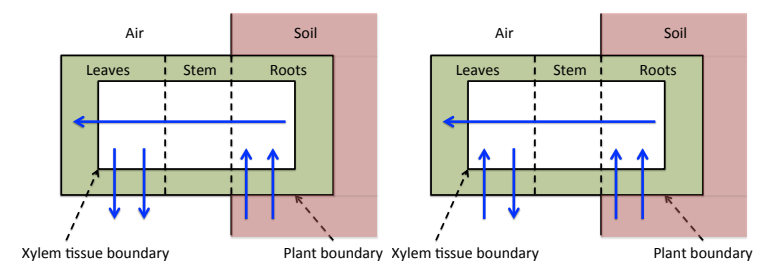

(a) Positive strong UDF

(b) Positive weak UDF

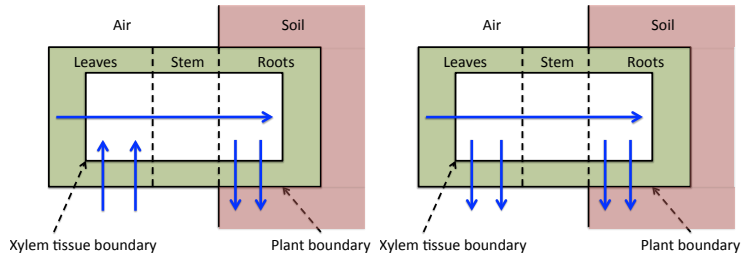

(c) Negative strong UDF (d) Negative weak UDF

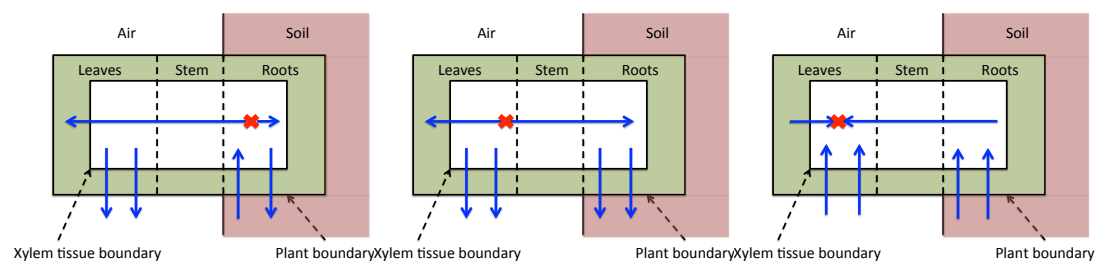

(e) Divergent root MDF (f) Divergent leaf MDF (g) Convergent leaf MDF

Figure 11: Unidirectional flow (UDF) and Multidirectional flow (MDF) classifications. The solid arrows indicate the direction of flows, and the cross indicates either the point of flow divergence or convergence. 
in the leaves $\left(p^{e x t}\right)$ equalling or not equalling the pressure at $z=0\left(p^{0}\right)$, with and without the effect of gravity $(G)$. The categories of regimes for the cases are listed in Table 3. The case of $p^{e x t}=p^{0}$ and $G=0$ is the simplest and yields only one critical relationship of biological interest; $M_{\text {crit }}^{L}$, and 3 possible flow regimes, positive UDF and divergent and convergent leaf MDF. $M_{\text {crit }}^{L}$ provides a critical relationship between the ratio of radial to axial leaf conductivity and the ratio of leaf to plant length, which persists for all the cases considered.

The inclusion of gravity causes the critical $M^{L}$ to couple to the value of $p^{0}$ (and hence $p^{e x t}$ ), and varying $p^{0}$ leads to 5 possible flow regimes, for which we have provided the bounds. The optimum flow regime for nutrient transport to the leaves is positive strong UDF, which occurs when $p^{0} \ll p^{\text {soil }}$. For smaller magnitudes of $p^{0}$ the flow can become multidirectional which at most seems unphysical, and at least seems detrimental to nutrient transport, and plant survival. The regime between positive strong UDF and divergent

\begin{tabular}{|c|c|c|}
\hline \multicolumn{3}{|c|}{$p^{0}=p^{e x t}$} \\
\hline$G=0$ & $\begin{array}{l}\text { positive strong UDF } \\
\text { divergent leaf MDF } \\
\text { convergent leaf MDF }\end{array}$ & $\begin{array}{l}M^{L}<M_{c r i t}^{L} \\
M^{L} \gtrsim M_{c r i t}^{L} \\
M^{L}>M_{c r i t}^{L}\end{array}$ \\
\hline$G \neq 0$ & $\begin{array}{l}\text { positive strong UDF } \\
\text { divergent root MDF } \\
\text { divergent leaf MDF } \\
\text { negative weak UDF } \\
\text { negative strong UDF } \\
\end{array}$ & $\begin{array}{l}p^{0}<\left.p_{\text {crit }}^{0 R}\right|_{z=1} ^{0} \\
\left.p_{\text {crit }}^{0 R}\right|_{z=1}<p^{0}<p_{\text {crit }}^{0 S} \\
p_{\text {crit }}^{0 S}<p^{0}<\left.p_{\text {crit }}^{0 L}\right|_{z=0} \\
\left.p_{\text {crit }}^{0 L}\right|_{z=0}<p^{0}<p^{\text {soil }}-\frac{2 G e^{M^{R}\left(1+z_{2}\right)}}{\sqrt{M^{R}} \kappa_{1}} \\
p^{0}>p^{\text {soil }}-\frac{2 G e^{M^{R}\left(1+z_{2}\right)}}{\sqrt{M^{R}} \kappa_{K_{1}}}\end{array}$ \\
\hline \multicolumn{3}{|r|}{$p^{0} \neq p^{e x t}$} \\
\hline$G=0$ & $\begin{array}{l}\text { convergent leaf MDF } \\
\text { positive weak UDF } \\
\text { positive strong UDF } \\
\text { divergent leaf MDF }\end{array}$ & $\begin{array}{l}p^{0}<\left.p_{\text {crit }}^{0}\right|_{z=0} \\
\left.p_{\text {crit }}^{0}\right|_{z=0}<p^{0}<p^{e x t} \\
p^{e x t}<p^{0}<\left.p_{\text {crit }}^{0}\right|_{z=z_{1}} \\
p^{0}>\left.p_{\text {crit }}^{0}\right|_{z=z_{1}}\end{array}$ \\
\hline$G \neq 0$ & $\begin{array}{l}\text { convergent leaf MDF } \\
\text { positive weak UDF } \\
\text { positive strong UDF } \\
\text { divergent root MDF } \\
\text { divergent leaf MDF }\end{array}$ & $\begin{array}{l}p^{0}<\left.p_{\text {crit }}^{0}\right|_{z=0} \\
\left.p_{\text {crit }}^{0}\right|_{z=0}<p^{0}<p^{e x t} \\
p^{\text {ext }}<p^{0}<\left.p_{\text {crit }}^{0}\right|_{z=1} \\
\left.p_{\text {crit }}^{0}\right|_{z=1}<p^{0}<\left.p_{\text {crit }}^{0}\right|_{z=z_{1}} \\
p^{0}>\left.p_{\text {crit }}^{0}\right|_{z=z_{1}}\end{array}$ \\
\hline
\end{tabular}

Table 3: Summary of the flow regimes for the cases of: xylem pressure at $z=0$ equals leaf air-space pressure $\left(p^{0}=p^{\text {ext }}\right)$, and for differing xylem pressure at $z=0$ and leaf air-space pressure $\left(p^{0} \neq p^{e x t}\right)$. UDF represents unidirectional flow, MDF represents multidirectional flow.

leaf MDF is divergent root MDF. This regime seems somewhat physical as there is evidence that similar flows occur; for example passive water efflux from roots to soil of lower water potential has been observed and is known as hydraulic lift (Caldwell et al., 1998). Water is released from roots when transpiration is low, which is usually at night, and then reabsorbed the next 
day and transpired. This agrees with the findings in this paper; i.e. that an increase in $p^{0}$ (and $p^{e x t}$ ), which occurs when transpiration is reduced, results in $\mathrm{MDF}$ in the root zone and consequently water efflux into the soil. The traditional case of hydraulic lift discussed by Caldwell et al. (1998) is characterised by outwards radial flow from the roots to the soil in the upper soil layers and inwards radial flow at the lower soil layers, which is opposite to the profile found in this paper for root MDF. This traditional profile explains why the process is called hydraulic lift, as water is transferred from lower moist soil to higher dry soil. However, the opposite flow profile (inwards radial flow at the upper soil layers and outwards radial flow in the lower soil layers) which is called inverse hydraulic lift is also found to occur, but usually for roots grown in dry soils (Caldwell et al., 1998).

Inverse hydraulic lift displays the same radial water profile as the root MDF seen in this paper, although the process may be different, since inverse hydraulic lift involves the transfer of water downwards which is not seen in the divergent flow of the root UDF. However, this may be explained by the fact that soils possess a gradient of water potential with depth, whereas in this paper a constant value for soil pressure was used. Therefore including a more physical soil potential gradient into the model may improve the results described here and allow a closer comparison to experimental results.

The case that $p^{0} \neq p^{e x t}$ was considered due to the inhomeogenity of the leaf air-spaces which could lead to a profile of varying potentials. Even without the effects of gravity, this case resulted in 4 flow regimes; positive UDF encompassed by MDF regimes, indicating that only a narrow deviation of $p^{0}$ from $p^{e x t}$ would allow UDF. Similarly as to the case $p^{0}=p^{e x t}$, the inclusion of gravity caused the addition of a flow regime (divergent root MDF), primarily due to the change of $p_{\text {crit }}^{0}$ equalling a constant in the root zone to $p_{c r i t}^{0}$ varying in the root zone. For all cases, we have provided the relationship between the parameter values that would give rise to unidirectional flow and hence optimum nutrient transport.

The transport of nutrient in the xylem vessel is primarily due to advection within the transpiration stream. We however, have also included diffusive transport due to the fact that diffusion can become significant near the vessel boundaries, or at night when the transpirational flow reduces. In addition, diffusion allows the modelling of an axial flux of nutrient through the xylem termini in the roots which may occur due to the nutrient sink effect of the growing root meristemic tissue. We have solved the model analytically for large $P e$ and numerically for all $P e$, and found very good agreement between the analytical and numerical models for high $P e$ which is applicable to the xylem vessels since $P e \approx 10^{7}$. We model the flow at night time by considering the effect of increased $p^{e x t}$ on the flow, and see that as $p^{0}$ approaches the value of $p^{\text {soil }}$, the water flow reduces, which consequently increases the contribution of diffusion to nutrient transport. Note that increasing $p^{e x t}$ also represents the environmental condition of high humidity. 
In addition, we have seen that an axial nutrient flux $f$ has a large effect on nutrient transport, especially in the root zone. This flux has not been observed in the literature, and hence its physical value is unknown. However, experiments can be carried out measuring the profile of phosphate, or other radio-labelled compounds, within the xylem vessel close to xylem tips to see if any boundary layer effects occur. This model therefore, in conjunction with experimental data can be used to determine firstly whether $f$ occurs, and secondly if it is of order $P e^{-\frac{1}{2}}$. Similarly, the functional forms of the loading/unloading parameter $F$ can be estimated by combining with experiments.

\section{Conclusions and Future work}

The aim of this work was to investigate the transport of water and nutrient within the xylem vessels from roots to leaves. We have seen that different water flow regimes are possible; some of which are unphysical, but some have been observed in nature such as positive unidirectional flow, and divergent root multidirectional flow which is similar to hydraulic lift. The positive unidirectional flow regime represents the optimum regime for nutrient transport and we have provided bounds for this for all cases considered. In addition, we have solved for the transport of phosphate considering convective and diffusive transport, and have seen that diffusion can be significant. This model only addresses one aspect of the fertilser-to-crop-yield transport pathway; and in order for this model to help improve efficiency of this pathway, it should be coupled to models of root growth and water and nutrient uptake, leaf and grain growth models, thus creating a whole plant model which can be used for optimisation and determination of plant parameters.

\section{Acknowledgements}

We thank Helena Unwin for conducting a literature review on xylem vessel sizes in wheat. This work was sponsored by Defra, BBSRC (BB/J000868/1), Scottish Government, AHDB, and other industry partners through Sustainable Arable LINK Project LK09136 and the BBSRC (BB/I024283/1). Tiina Roose is funded by The Royal Society University Research Fellowship.

\section{References}

J. L. Araus, L. Alegre, L. Tapia, and R. Calafell, (1986), Relationship between leaf structure and gas exchange in wheat leaves at different insertion levels, J. Exp. Bot. 37(9), 1323-1333. 
E. Bennett, and J. Elser, (2011), A broken biogeochemical cycle, Nature 478.

I. J. Bingham, and E. A. Stevenson, (1993), Control of root growth: effects of carbohydrates on the extension, branching and rate of respiration of different fractions of wheat roots, Physiologia Plantarum 88(1), 149-158.

G. D. Bowen, (1970), Effects of soil temperature on root growth and on phosphate uptake along Pinus radiata roots, Soil Research 8(1), 31-42.

M. Bucher, (2007), Functional biology of plant phosphate uptake at root and mycorrhiza interfaces, New Phytologist 173 (1), 11-26.

M. M. Caldwell, T. E. Dawson, and J. H. Richards, (1998), Hydraulic lift: consequences of water efflux from the roots of plants, Oecologia 113(2), 151-161.

N. A. Campbell (2008) in Campbell (9th Edition) Biology, Pearson/Benjamin Cummings, San Francisco; London, Chapter 36 Resource acquisition and transport in vascular plants, 764-784.

D. Cordell, J. O. Drangert, and S. White, (2009), The story of phosphorus: Global food security and food for thought, Global Environmental Change 19(2), 292-305.

H. H. Dixon, and J. Joly, (1895), On the ascent of sap, Philos Trans R Soc Lond Ser B. 186, 563-576.

C. Foyer, and C. Spencer, (1986), The relationship between phosphate status and photosynthesis in leaves. Planta 167(3), 369-375.

J. Frensch, and E. Steudle, (1989), Axial and radial hydraulic resistance to roots of maize (Zea mays L.), Plant Physiol. 91(2), 719-726.

A. Gallet, R. Flisch, J. P. Ryser, E. Frossard, and S. Sinaj, (2003), Effect of phosphate fertilization on crop yield and soil phosphorus status. Journal of Plant Nutrition and Soil Science 166(5), 568-578.

E. J. Hinch, (1991), Perturbation methods Vol(6), Cambridge University Press, Cambridge.

K. H. Jensen, K. Berg-Srensen, S. M. Friis, and T. Bohr, (2012), Analytic solutions and universal properties of sugar loading models in Münch phloem flow, J. Theor. Biol, 304, 286-296.

K. H. Jensen, J. Lee, T. Bohr, H. Bruus, N. M. Holbrook, and M. A, Zwieniecki, (2011), Optimality of the Münch mechanism for translocation of sugars in plants, J. R. Soc. Interface 8(61), 1155-1165. 
H. Jones, A. D. Tomos, R. A. Leigh, and R. G. W Jones, (1983), Waterrelation parameters of epidermal and cortical cells in the primary root of Triticum aestivum L., Planta 158(3), 230-236.

P. J. Kramer and J. S. Boyer, (1995), Water relations of plants and soils, Academic Press, San Diego, Chapter 7, 201-256.

L. Kutschera, E. Lichtenegger, and M. Sobotik, (2009), Wurzelatlas der Kulturpflanzen gemigter Gebiete mit Arten des Feldgemsebaues DLG Verlag, Frankfurt.

J. J. Landsberg, and N. D. Fowkes, (1978), Water movement through plant roots, Ann Bot. 42(3), 493-508.

F. J. Molz, (1981), Models of water transport in the soil-plant system: A review, Water Resources Research 17(5), 1245-1260.

J. Percival, (1921), The wheat plant, Duckworth and Co, London.

R. H. Perry, and D. W. Green, (1997), Perry's chemical engineers' handbook 7th Ed., McGraw Hill, New York.

E. Steudle, (2001), The cohesion-tension mechanism and the acquisition of water by plant roots, Annu. Rev. Plant Physiol Plant Mol Biol. 52(1), 847-875.

E. Steudle, and C.A. Peterson, (1998), How does water get through roots?, J. Exp. Bot. 49(322), 775-788.

M. V. Thompson, and N. M. Holbrook, (2003), Application of a single-solute non-steady-state phloem model to the study of long-distance assimilate transport, J. Theor. Biol, 220(4), 419-455.

M. T. Tyree, (1997), The Cohesion-Tension theory of sap ascent: current controversies, J. Exp. Bot. 48(10), 1753-1765. 


\section{Appendix A: Xylem dimensions in roots and leaves}

\begin{tabular}{|c|c|c|c|c|}
\hline Type of vessel & average $\hat{a}(\mu \mathrm{m})$ & $\hat{v}$ & $\hat{k}_{z}\left(\mathrm{~m}^{4} \mathrm{~Pa}^{-1} \mathrm{~s}^{-1}\right)$ & $1 / \mathrm{Pe}$ \\
\hline Single large pitted & 25 & $1 \times 1$ & $1.72 \times 10^{-16}$ & $1.14 \times 10^{-8}$ \\
centripetal & 8.75 & $1 \times 7.5$ & $1.94 \times 10^{-17}$ & $9.3 \times 10^{-8}$ \\
protoxylem & 3.75 & $2 \times 7.5$ & $1.31 \times 10^{-18}$ & $5.06 \times 10^{-7}$ \\
\hline & & & $\sum=1.93 \times 10^{-16}$ & $\sum=6.1 \times 10^{-7}$ \\
\hline
\end{tabular}

Table 4: Calculations of $\hat{k}_{z}$ and $1 / P e$ in the root zone based on number and radii of xylem vessels in wheat from Percival (1921) (pg 39-40). The number of vessels $\hat{v}$ is given by number of vessels per bundle multiplied by the number of bundles.

\begin{tabular}{|c|c|c|c|c|}
\hline Leaf position & average $\hat{a}(\mu \mathrm{m})$ & $\hat{v}$ & $\hat{k}_{z}\left(\mathrm{~m}^{4} \mathrm{~Pa}^{-1} \mathrm{~s}^{-1}\right)$ & $1 / \mathrm{Pe}$ \\
\hline 5th or uppermost & 10 & $4 \times 13$ & $2.29 \times 10^{-16}$ & $7.13 \times 10^{-8}$ \\
4th & 10 & $4 \times 13$ & $2.29 \times 10^{-16}$ & $7.13 \times 10^{-8}$ \\
3rd & 10 & $4 \times 13$ & $2.29 \times 10^{-16}$ & $7.13 \times 10^{-8}$ \\
2nd & 10 & $4 \times 9$ & $1.59 \times 10^{-16}$ & $7.11 \times 10^{-8}$ \\
1st & 10 & $4 \times 9$ & $1.59 \times 10^{-16}$ & $7.11 \times 10^{-8}$ \\
\hline & & & average $=2.01 \times 10^{-16}$ & average $=7.12 \times 10^{-8}$ \\
\hline
\end{tabular}

Table 5: Calculations of $\hat{k}_{z}$ and $1 / P e$ in the leaf zone based on number and radii of xylem vessels in the stout bundles of wheat from Percival (1921) (pg 57-59). The number of vessels $\hat{v}$ is given by number of vessels per bundle multiplied by the number of bundles.

\section{Appendix B: Range of $p^{0}$ that would give rise to unidirectional flow for $p^{0} \neq p^{e x t}$, and $G=0$}

$$
\begin{gathered}
p_{\mid z=0}^{0 L}=p^{e x t}+\frac{\sqrt{M^{R}}\left(p^{\text {soil }}-p^{e x t}\right) \kappa_{1}}{f\left(z_{1}, z_{2}\right)}+\frac{G}{f\left(z_{1}, z_{2}\right)}\left(-2 e^{\sqrt{M^{R}}\left(1+z_{2}\right)}\right. \\
\left.+\left(\kappa_{2}+\sqrt{M^{R}}\left(z_{1}-z_{2}\right) \kappa_{1}\right) \cos \left(\sqrt{M^{L}} z_{1}\right)-\frac{\sqrt{M^{R}} \kappa_{1} \sin \left(\sqrt{M^{L}} z_{1}\right)}{\sqrt{M^{L}}}\right) \\
p_{\text {crit } \mid z=1}^{0 R}=p^{e x t}+\cos \left(\sqrt{M^{L}} z_{1}\right)\left(p^{\text {soil }}-p^{e x t}\right)+ \\
\begin{array}{c}
\frac{G e^{-\sqrt{M^{R}}}\left(z_{2}+1\right)}{2}\left(\cos \left(\sqrt{M^{L}} z_{1}\right)\left(\frac{\kappa_{1}}{\sqrt{M^{R}}}+\kappa_{2}\left(z_{1}-z_{2}\right)\right)-\frac{\kappa_{2} \sin \left(\sqrt{M^{L}} z_{1}\right)}{\sqrt{M^{L}}}\right) \\
\text { where } f\left(z_{1}, z_{2}\right)=\sqrt{M^{R}} \kappa_{1} \cos \left(\sqrt{M^{L}} z_{1}\right) \\
+\sqrt{M^{L}}\left(\kappa_{2}+\sqrt{M^{R}}\left(z_{1}-z_{2}\right) \kappa_{1}\right) \sin \left(\sqrt{M^{L}} z_{1}\right) .
\end{array}
\end{gathered}
$$




\section{Appendix C: Analytical solution for nutrient trans- port by convection and diffusion}

We seek an analytical solution for the transport of phosphate, $n$, due to both convection and diffusion, but consider that diffusion is only important near the boundaries $z=0, z_{1}, z_{2}, 1$. In the main bulk, convection dominates and we can neglect diffusion, but near the boundaries we expect the presence of boundary layers as the solution rapidly adjusts to the boundary conditions. For an introduction into boundary layer methods see Hinch (1991). For simpler analytical progress we neglect the effect of gravity as its effect is small, and as an example assume that $F$ is constant.

\section{Root zone}

We first consider the root zone and the boundary near $z=1$. We let $\epsilon=1 / P e$, which is small, such that the nutrient transport equation and boundary condition becomes

$$
\begin{aligned}
& \frac{d}{d z}\left(n^{R}\left(\frac{d p^{R}}{d z}-G\right)+\epsilon \frac{d n^{R}}{d z}\right)=-F^{R}, \\
& \epsilon \frac{d n^{R}}{d z}=-f \quad \text { at } z=1 .
\end{aligned}
$$

We scale $n^{R}=\epsilon^{-1} f \bar{n}^{R}$, and integrate (C.1) to give

$$
\begin{aligned}
& \epsilon^{-1} \bar{n}^{R}\left(\frac{d p^{R}}{d z}-G\right)+\frac{d \bar{n}^{R}}{d z}=-\frac{F^{R}}{f} z+k_{1}, \\
& \frac{d \bar{n}^{R}}{d z}=-1 \text { at } z=1,
\end{aligned}
$$

where $k_{1}$ is determined from the boundary condition (C.4) to be $\frac{F^{R}-f}{f}$. We define the boundary layer coordinate $x$ and let $1-z=\epsilon^{\alpha} x$, such that

$$
\begin{aligned}
& \epsilon^{-1} \bar{n}^{R} \tilde{R}_{1}-\epsilon^{-\alpha} \frac{d \bar{n}^{R}}{d x}=-\frac{F^{R}}{f}\left(1-\epsilon^{\alpha} x\right)+\frac{F^{R}-f}{f}, \\
& \epsilon^{-\alpha} \frac{d \bar{n}^{R}}{d x}=1 \text { at } x=0
\end{aligned}
$$


where $\tilde{R}_{1}$ is $\frac{d p^{R}}{d z}-G$ as a function of $\left(1-\epsilon^{\alpha} x\right)$ which we expand as follows

$$
\begin{aligned}
\tilde{R}_{1} & =\sqrt{M^{R}}\left(A_{5} e^{\sqrt{M^{R}} z}-A_{6} e^{-\sqrt{M^{R}} z}\right)-G \\
& =\sqrt{M^{R}}\left(\left(A_{5} e^{\sqrt{M^{R}}}-A_{6} e^{-\sqrt{M^{R}}}\right)\left(1+\frac{M^{R} \epsilon^{2 \alpha} x^{2}}{2 !}+O\left(\epsilon^{4 \alpha}\right)\right)\right. \\
& \left.-\left(A_{5} e^{\sqrt{M^{R}}}+A_{6} e^{-\sqrt{M^{R}}}\right)\left(\sqrt{M^{R}} \epsilon^{\alpha} x+\frac{M^{R} \frac{3}{2} \epsilon^{3 \alpha} x^{3}}{3 !}+O\left(\epsilon^{5 \alpha}\right)\right)\right) \\
& -G .
\end{aligned}
$$

Setting $G=0$, we find that $A_{5} e^{\sqrt{M^{R}}}-A_{6} e^{-\sqrt{M^{R}}}=0$ and

$$
\begin{aligned}
& A_{5} e^{\sqrt{M^{R}}}+A_{6} e^{-\sqrt{M^{R}}}= \\
& \frac{2 \sqrt{M^{L}} e^{\sqrt{M^{R}}}\left(z_{2}+1\right)\left(p^{e x t}-p^{0}+\cos \left(\sqrt{M^{L}} z_{1}\right)\left(p^{\text {soil }}-p^{e x t}\right)\right)}{\sqrt{M^{R}} \kappa_{1} \sin \left(\sqrt{M^{L}} z_{1}\right)-\sqrt{M^{L}}\left(\sqrt{M^{R}}\left(z_{1}-z_{2}\right) \kappa_{1}+\kappa_{2}\right) \cos \left(\sqrt{M^{L}} z_{1}\right)},
\end{aligned}
$$

which is negative for the parameter values considered (values of $M^{L}$ below that given by Equation 22). Therefore, with $G=0$, we find

$$
\tilde{R}_{1}=-\sqrt{M^{R}}\left(A_{5} e^{\sqrt{M^{R}}}+A_{6} e^{-\sqrt{M^{R}}}\right)\left(\sqrt{M^{R}} \epsilon^{\alpha} x+\frac{M^{R} \frac{3}{2} \epsilon^{3 \alpha} x^{3}}{3 !}+O\left(\epsilon^{5 \alpha}\right)\right),
$$

which we rewrite as $\tilde{R}_{1}=\tilde{R}_{11} \epsilon^{\alpha} x+\tilde{R}_{13} \epsilon^{3 \alpha} x^{3}+O\left(\epsilon^{5 \alpha}\right)$, and whose coefficients are positive and are given by

$$
\begin{aligned}
& \tilde{R}_{11}=-M^{R}\left(A_{5} e^{\sqrt{M^{R}}}+A_{6} e^{-\sqrt{M^{R}}}\right), \\
& \tilde{R}_{13}=-\frac{M^{R^{2}}}{3 !}\left(A_{5} e^{\sqrt{M^{R}}}+A_{6} e^{-\sqrt{M^{R}}}\right) .
\end{aligned}
$$

Note that if $G \neq 0$ then $A_{5} e^{\sqrt{M^{R}}}+A_{6} e^{-\sqrt{M^{R}}}=G / \sqrt{M^{R}}$ and hence the series would be become $\tilde{R}_{1}=\tilde{R}_{11} \epsilon^{\alpha} x+G \frac{M^{R} \epsilon^{2 \alpha} x^{2}}{2 !}+\tilde{R}_{13} \epsilon^{3 \alpha} x^{3}+O\left(\epsilon^{4 \alpha}\right)$ which makes analytical progress more complicated. Therefore, with $G=0$, Equation (C.5) becomes

$\epsilon^{-1} \bar{n}^{R}\left(\tilde{R}_{11} \epsilon^{\alpha} x+\tilde{R}_{13} \epsilon^{3 \alpha} x^{3}+O\left(\epsilon^{5 \alpha}\right)\right)-\epsilon^{-\alpha} \frac{d \bar{n}^{R}}{d x}=-\frac{F^{R}}{f}\left(1-\epsilon^{\alpha} x\right)+\frac{F^{R}-f}{f}$. 
In order for the viscous terms to balance the convective terms, we choose $\alpha=\frac{1}{2}$ which leads to

$$
\begin{aligned}
& \bar{n}^{R}\left(\tilde{R}_{11} x+\tilde{R}_{13} \epsilon x^{3}+O\left(\epsilon^{2}\right)\right)-\frac{d \bar{n}^{R}}{d x}=-\frac{F^{R}}{f}\left(\epsilon^{\frac{1}{2}}-\epsilon x\right)+\left(\frac{F^{R}-f}{f}\right) \epsilon^{\frac{1}{2}}, \\
& \frac{d \bar{n}^{R}}{d x}=\epsilon^{\frac{1}{2}} \text { at } x=0 .
\end{aligned}
$$

We expand $\bar{n}^{R}=\bar{n}_{0}^{R}+\epsilon^{\frac{1}{2}} \bar{n}_{1}^{R}+\epsilon \bar{n}_{2}^{R}+O\left(\epsilon^{\frac{3}{2}}\right)$ and solve Equation (C.13) at successive orders of $\epsilon$ subject to the boundary condition (C.14). The $O\left(\epsilon^{0}\right)$ terms of Equations (C.13) and (C.14) are

$$
\begin{aligned}
& \bar{n}_{0}^{R} \tilde{R}_{11} x-\frac{d \bar{n}_{0}^{R}}{d x}=0, \\
& \frac{d \bar{n}_{0}^{R}}{d x}=0 \text { at } x=0,
\end{aligned}
$$

which has the solution $\bar{n}_{0}^{R}=C_{0} e^{\frac{1}{2} \tilde{R}_{11} x^{2}}$, where $C_{0}$ is a free constant found by matching to the bulk solution. Since this solution grows as $x$ increases, the only choice of $C_{0}$ is $C_{0}=0$ such that $\bar{n}_{0}^{R}=0$.

The $O\left(\epsilon^{\frac{1}{2}}\right)$ terms of Equations (C.13) and (C.14) are

$$
\begin{aligned}
& \bar{n}_{1}^{R} \tilde{R}_{11} x-\frac{d \bar{n}_{1}^{R}}{d x}=-\frac{F^{R}}{f}+\frac{F^{R}-f}{f}, \\
& \frac{d \bar{n}_{1}^{R}}{d x}=1 \text { at } x=0,
\end{aligned}
$$

which has the solution $\bar{n}_{1}^{R}=\left(\frac{1}{2} \sqrt{\frac{2 \pi}{\tilde{R}_{11}}} \operatorname{erf}\left(\frac{1}{2} \sqrt{2 \tilde{R}_{11}} x\right)+C_{1}\right) e^{\frac{1}{2} \tilde{R}_{11} x^{2}}$, where $C_{1}$ is a free constant found by matching to the bulk solution. To ensure a non-growing solution, we choose $C_{1}=-\frac{1}{2} \sqrt{\frac{2 \pi}{\tilde{R}_{11}}}$, such that

$$
\bar{n}_{1}^{R}=\frac{1}{2} \sqrt{\frac{2 \pi}{\tilde{R}_{11}}} e^{\frac{1}{2} \tilde{R}_{11} x^{2}}\left(\operatorname{erf}\left(\frac{1}{2} \sqrt{2 \tilde{R}_{11} x}\right)-1\right) .
$$

The $O(\epsilon)$ terms of Equations (C.13) and (C.14) are

$$
\begin{array}{ll}
\bar{n}_{2}^{R} \tilde{R}_{11} x-\frac{d \bar{n}_{2}^{R}}{d x}=\frac{F^{R}}{f} x, \\
\frac{d \bar{n}_{2}^{R}}{d x}=0 \quad \text { at } x=0,
\end{array}
$$

which has the solution $\bar{n}_{2}^{R}=\frac{F^{R}}{\tilde{R}_{11} f}+C_{2} e^{\frac{1}{2} \tilde{R}_{11} x^{2}}$ where $C_{2}$ is a free constant found by matching to the bulk solution. To ensure a non-growing solution 
we choose $C_{2}=0$, such that $\bar{n}_{2}^{R}=\frac{F^{R}}{\tilde{R}_{11} f}$. A summary of the boundary layer solution near $z=1$ is

$$
n^{R}=\epsilon^{-1} f\left(\epsilon^{\frac{1}{2}} \bar{n}_{1}^{R}+\epsilon \bar{n}_{2}^{R}+O\left(\epsilon^{\frac{3}{2}}\right)\right)=f\left(\epsilon^{-\frac{1}{2}} \bar{n}_{1}^{R}+\epsilon^{0} \bar{n}_{2}^{R}+O\left(\epsilon^{\frac{1}{2}}\right)\right) .
$$

The boundary layer solution has to match to the bulk solution which is governed by

$$
n^{R}\left(\frac{d p^{R}}{d z}\right)+\epsilon \frac{d n^{R}}{d z}=-F^{R} z+C_{4}
$$

where $C_{4}$ is an unknown constant. We similarly let $n^{R}=\epsilon^{0} n_{0}^{R}+O\left(\epsilon^{\frac{1}{2}}\right)$ and find $n_{0}^{R}=\frac{-F^{R} z+C_{4}}{\frac{d p}{d z}}$ which is equivalent to the convection-only solution. Matching the bulk to the boundary layer solution we find that $\bar{n}_{1}$ matches to zero, and $\bar{n}_{2}$ matches to the bulk solution to give $C_{4}=F^{R}$. Following a similar procedure near the stem-root boundary, we find that the boundary layer solution near $z=z_{2}$ is

$$
n_{0}^{R}=k_{1} e^{-\tilde{R}_{20} P e\left(z-z_{2}\right)}+\frac{F^{R}\left(1-z_{2}\right)}{\tilde{R}_{20}},
$$

where $\tilde{R}_{20}=\sqrt{M^{R}}\left(A_{5} e^{\sqrt{M^{R}}} z_{2}-A_{6} e^{-\sqrt{M^{R}} z_{2}}\right)$ and $k_{1}$ is an unknown constant. Therefore the composite solution in root is

$$
\begin{aligned}
& n^{R}=\frac{F^{R}(1-z)}{\frac{d p^{R}}{d z}}+k_{1} e^{-\tilde{R}_{20} P e\left(z-z_{2}\right)}+ \\
& f \sqrt{P e} \frac{1}{2} \sqrt{\frac{2 \pi}{\tilde{R}_{11}}} e^{\frac{1}{2} \tilde{R}_{11} P e(1-z)^{2}}\left(\operatorname{erf}\left(\frac{1}{2} \sqrt{2 \tilde{R}_{11} P e}(1-z)\right)-1\right)+O\left(\epsilon^{\frac{1}{2}}\right) .
\end{aligned}
$$

The constant $k_{1}$ will be determined by applying the continuity boundary conditions but first the solutions in the stem and leaf regions have to be calculated.

\section{Stem zone}

Since $p^{S}=A_{3} z+A 4$, the nutrient transport in the stem zone is governed by

$$
n^{S} A_{3}+\epsilon \frac{d n^{S}}{d z}=-F^{S} z+k_{2}
$$

where $k_{2}$ is an unknown constant. We expand $n^{S}=n_{0}^{S}+O\left(\epsilon^{\frac{1}{2}}\right)$ and seek boundary layer solutions near $z=z_{1}$ and $z=z_{2}$ to match to the central 
bulk region. We find that a boundary layer exists only near $z=z_{1}$ such that the composite solution in the stem region is given by

$$
n^{S}=\frac{-F^{S} z+k_{2}}{A_{3}}+k_{3} e^{-A_{3} P e\left(z-z_{1}\right)}+O\left(\epsilon^{\frac{1}{2}}\right),
$$

where $k_{3}$ is also an unknown constant, which together with $k_{2}$ will be determined from the continuity boundary conditions.

\section{Leaf zone}

The nutrient transport in the leaf zone is governed by

$$
n^{L}\left(\frac{d p^{L}}{d z}\right)+\epsilon \frac{d n^{L}}{d z}=-F^{L} z+k_{4},
$$

where $k_{4}$ is an unknown constant. We expand $n^{L}=n_{0}^{L}+O\left(\epsilon^{\frac{1}{2}}\right)$ and seek boundary layer solutions near $z=0$ and $z=z_{1}$ to match to the central bulk region. We find that a boundary layer exists only near $z=0$ where the boundary condition that $n=\phi$ at $z=0$ has to be satisfied. We solve for this boundary layer by defining a boundary layer variable $y$ and letting $z=\epsilon^{\beta} y$ such that

$$
n^{L} \tilde{L}+\epsilon^{1-\beta} \frac{d n^{L}}{d y}=-F^{L} \epsilon^{\beta} y+k_{4},
$$

where $\tilde{L}=\frac{d p^{L}}{d z}$ as a function of $\epsilon^{\beta} y$, which we expand as follows

$$
\begin{aligned}
\tilde{L} & =\sqrt{M^{L}}\left(A_{1} \cos \left(\sqrt{M^{L}} \epsilon^{\beta} y\right)-A_{2} \sin \left(\sqrt{M^{L}} \epsilon^{\beta} y\right)\right) \\
& =\sqrt{M^{L}}\left(A_{1}\left(1-\frac{M^{L} \epsilon^{2 \beta} y^{2}}{2 !}+O\left(\epsilon^{4 \beta}\right)\right)-A_{2}\left(\sqrt{M^{L}} \epsilon^{\beta} y+O\left(\epsilon^{3 \beta}\right)\right)\right) .
\end{aligned}
$$

We rewrite $\tilde{L}$ as $\tilde{L}=\tilde{L}_{1}+\epsilon^{\beta} y \tilde{L}_{2}+O\left(\epsilon^{2 \beta}\right)$, whose coefficients are positive and are given by

$$
\tilde{L}_{1}=A_{1} \sqrt{M^{L}}, \quad \tilde{L}_{2}=-A_{2} M^{L} .
$$

We expand $n^{L}=\epsilon^{0} n_{0}^{L}+O\left(\epsilon^{\frac{1}{2}}\right)$ and choose $\beta=1$ such that

$$
\begin{aligned}
& n^{L}\left(\tilde{L}_{1}+\epsilon y \tilde{L}_{2}+O\left(\epsilon^{2}\right)\right)+\frac{d n^{L}}{d y}=-F^{L} \epsilon y+k_{4}, \\
& n^{L}=\phi \quad \text { at } y=0 .
\end{aligned}
$$

The leading order terms of Equations (C.32) and (C.33) are

$$
\begin{aligned}
& n_{0}^{L} \tilde{L}_{1}+\frac{d n_{0}^{L}}{d y}=k_{4}, \\
& n_{0}^{L}=\phi \quad \text { at } y=0,
\end{aligned}
$$


which has the solution $n_{0}^{L}=\frac{k_{4}}{\tilde{L}_{1}}+\left(\phi-\frac{k_{4}}{\tilde{L}_{1}}\right) e^{-\tilde{L}_{1} y}$, and which matches to the bulk solution near $y=0$. The composite leaf solution is

$$
n^{L}=\frac{-F^{L} z+k_{4}}{\frac{d p^{L}}{d z}}+\left(\phi-\frac{k_{4}}{\tilde{L}_{1}}\right) e^{-\tilde{L}_{1} P e z}+O\left(\epsilon^{\frac{1}{2}}\right) .
$$

\section{Applying continuity boundary conditions}

The solutions (C.25), (C.27), and (C.36) represent the leading order composite solutions of $n$ in the root, stem and leaf zones respectively. The unknown constants $k_{1}, k_{2}, k_{3}$, and $k_{4}$ are determined by applying the continuity boundary conditions and retaining only the leading order terms; terms up to but not including those at $O\left(\epsilon^{\frac{1}{2}}\right)$. At leading order, the continuity of flux condition at $z=z_{1}$ reduces to

$$
-\tilde{L}_{1}\left(\phi-\frac{k_{4}}{\tilde{L}_{1}}\right) e^{-\tilde{L}_{1} P e z_{1}}=-A_{3} k_{3} .
$$

Since the terms on the left hand side are asymptotically zero, this results in $k_{3}=0$. Similarly, the continuity of flux condition at $z=z_{2}$ at leading order reduces to

$$
\begin{aligned}
& -A_{3} k_{3} e^{-A_{3} P e\left(z_{2}-z_{1}\right)}=-\tilde{R}_{20} k_{1}-f+ \\
& \frac{1}{2} f \sqrt{2 \pi \tilde{R}_{11} P e}\left(1-z_{2}\right) e^{\frac{1}{2} \tilde{R}_{11} P e\left(1-z_{2}\right)^{2}} \operatorname{erfc}\left(\frac{1}{2} \sqrt{2 \tilde{R}_{11} P e}\left(1-z_{2}\right)\right) .
\end{aligned}
$$

Since $k_{3}=0$, we find that

$k_{1}=\frac{f}{\tilde{R}_{20}}\left(\frac{\sqrt{2 \pi \tilde{R}_{11} P e}}{2}\left(1-z_{2}\right) e^{\frac{1}{2} \tilde{R}_{11} P e\left(1-z_{2}\right)^{2}} \operatorname{erfc}\left(\frac{1}{2} \sqrt{2 \tilde{R}_{11} P e}\left(1-z_{2}\right)\right)-1\right)$,

using the asymptotic structure of erfc we find that $k_{1}$ is also zero to leading order with a corrections at $O(1 / P e)$. Applying continuity of $n$ at $z=z_{1}$ and $z=z_{2}$, we find that the remaining constants to leading order are

$$
k_{2}=F^{R}\left(1-z_{2}\right)+F^{S} z_{2}+A_{3} \chi, \quad k_{4}=z_{1}\left(F^{L}-F^{S}\right)+k_{2}
$$

where $\chi=-\frac{f}{2} \sqrt{\frac{2 \pi P e}{\tilde{R}_{11}}} e^{\frac{1}{2}} \tilde{R}_{11} P e\left(1-z_{2}\right)^{2} \operatorname{erfc}\left(\frac{1}{2} \sqrt{2 \tilde{R}_{11} P e}\left(1-z_{2}\right)\right)$. 